Discussion Paper No. 11-008

Orphans at Risk in Sub-Saharan Africa:

Evidence on Educational and Health Outcomes

Katja Coneus and Andrea M. Mühlenweg

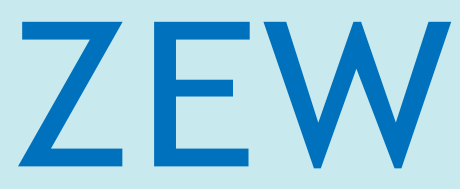

Zentrum für Europäische Wirtschaftsforschung $\mathrm{GmbH}$

Centre for European

Economic Research 
Discussion Paper No. 11-008

\section{Orphans at Risk in Sub-Saharan Africa: Evidence on Educational and Health Outcomes}

Katja Coneus and Andrea M. Mühlenweg

Download this ZEW Discussion Paper from our ftp server:

ftp://ftp.zew.de/pub/zew-docs/dp/dp11008.pdf

Die Discussion Papers dienen einer möglichst schnellen Verbreitung von neueren Forschungsarbeiten des ZEW. Die Beiträge liegen in alleiniger Verantwortung der Autoren und stellen nicht notwendigerweise die Meinung des ZEW dar.

Discussion Papers are intended to make results of ZEW research promptly available to other economists in order to encourage discussion and suggestions for revisions. The authors are solely responsible for the contents which do not necessarily represent the opinion of the ZEW. 


\section{Das Wichtigste in Kürze}

Die hohen Sterblichkeitsraten junger Erwachsener in vielen subsaharischen Ländern Afrikas gehen mit besonders hohen und steigenden Anteilen an Waisenkindern einher. In dieser Arbeit untersuchen wir die Auswirkung des Verlusts eines Elternteils auf die Bildung und gesundheitliche Entwicklung der Kinder. Diese Untersuchung basiert auf vergleichbaren Querschnittsdaten für elf Länder des subsaharischen Afrikas. Als Waisenkinder bezeichnen wir Kinder, die mindestens einen biologischen Elternteil verloren haben. In den betrachteten Ländern sind etwa 10 \% oder mehr der unter fünfzehnjährigen Kinder in privaten Haushalten Waisenkinder. Entsprechend ist es sehr wahrscheinlich, dass die hohe Elternsterblichkeit nicht nur die demographische Zusammensetzung sondern auch die Humankapitalbildung der Länder verändert. Um einen Einblick in diese Veränderung zu erhalten, analysieren wir deshalb aus der Mikroperspektive, inwiefern der Verlust eines Elternteils die schulische und gesundheitliche Entwicklung von Kindern beeinflusst. Unsere Analyse zielt darauf ab, Kinder in möglichst gleichen Kontexten und sonstigen Umständen zu vergleichen. Wir berücksichtigen auch den Einfluss verschiedener Familienstrukturen und vergleichen Waisenkinder mit Kindern, die keine Waisenkinder sind, die aber dennoch ohne mindestens einen biologischen Elternteil aufwachsen. Unsere Schätzergebnisse zeigen, dass Waisenkinder eine geringere Bildung sowie tendenziell eine schlechtere gesundheitliche Entwicklung aufweisen als vergleichbare Nicht-Waisen. Die kumulative Dauer des Schulbesuchs ist für die Waisenkinder im Vergleich zu Nicht-Waisen reduziert. Dieser Effekt liegt durchschnittlich zwischen einem fünftel und einem halben Schuljahr. Die negativen Effekte sind besonders stark, wenn die Kinder ohne ihre biologische Mutter aufwachsen, während wir kaum einen zusätzlichen Einfluss des biologischen Vaters beobachten. Diese Ergebnisse legen Nahe, dass es weitere Erfordernisse für Entwicklungspolitik gibt, die sich der Situation von Waisenkindern im subsaharischen Afrika annimmt. Insbesondere sollte die Bildung von Kindern stärker gefördert werden, die ohne eine Mutter aufwachsen, und zwar auch dann, wenn diese Mütter nicht verstorben sind. 


\section{Non Technical Summary}

Mortality rates among prime-age adults are dramatically high in many sub-Saharan African countries, and, as a consequence, the number of orphaned children is high and increasing. In this paper, we identify the educational and health effects of orphanhood in sub-Saharan African countries. We draw on comparable data for eleven sub-Saharan African countries with especially high rates of orphanhood. We define an orphan as a child with at least one deceased biological parent. In the analyzed countries, $10 \%$ or more of children under the age of 15 growing up in private households are orphans. It is very likely that the high incidence of parental death not only impacts the demographic composition of the respective countries, but also affects human capital formation. Therefore, on the micro level, we seek to examine how being an orphan affects individual schooling and health outcomes. More specifically, our research compares the educational and health outcomes of orphans living under the same conditions as non-orphans. We also examine the impacts of various family structures and compare social orphans (non-orphaned children not living with a biological parent) to orphans. In sum, our findings suggest that in most countries, orphans and social orphans growing up under the same conditions as non-orphans are significantly worse off in terms of their observed educational and health outcomes. Compared to children whose parents are alive and live with their biological parents, orphans lag behind in education. Our estimations indicate that orphaned children lag behind their non-orphan counterparts in cumulative school participation by onefifth to one-half of a year. Children are especially harmed by not growing up with a biological mother. In most countries, we observe hardly any additional effect of growing up without a father. The findings call for policies that specifically address the situation faced by orphans in sub-Saharan African countries. According to our results, policy measures should seek to specifically assist children whose mothers are alive, but absent from the household. 


\title{
Orphans at Risk in Sub-Saharan Africa: Evidence on Educational and Health Outcomes
}

\author{
Katja Coneus and Andrea M. Mühlenweg \\ Centre for European Economic Research (ZEW) Mannheim \\ ZEW Mannheim \\ L7, 1 \\ D-68161 Mannheim \\ Germany \\ Phone: $++49-621-1235-280$
}

\begin{abstract}
In this paper, we examine how orphanhood affects children's educational and health outcomes in eleven sub-Saharan African countries. Our analysis is based on a comparison of orphans and non-orphaned children living under the same conditions. We also examine the impacts of various family structures and compare social orphans (non-orphaned children not living with a biological parent) to orphans. Using household fixed-effects estimation, we provide evidence that children not living with a biological parent lag behind in education and are more often malnourished and stunted. Educational gaps are particularly evident among orphans and social orphans not living with a mother. The effect of paternal death or absence is rather modest in most countries.
\end{abstract}

JEL Classification: I12, I21, J24

Keywords: orphans, family structure, human capital, sub-Saharan Africa, fixed-effects

Acknowledgements: We are especially grateful to Irene Bertschek, Stephan Dlugosz, Bernd Fitzenberger, Markus Frölich, Juanna Joensen, David Ribar, Analia Schlosser and Anthony Strittmatter for fruitful discussions and valuable comments on earlier versions of this work. Thanks to Eric Dubiel for excellent research assistance. Also, we thank Jan Kinne and Helena Stroh for research assistance in generating the geographical maps. We thank participants of the Interdisciplinary Symposium on Skill Formation in Childhood and Adolescence at the University of Freiburg and seminar participants at ZEW for valuable comments. Andrea Mühlenweg thanks colleagues at the Economics Department of the University of North Carolina at Greensboro for providing a stimulating research environment and many interesting discussions during her research visit. 



\section{Introduction}

Mortality rates among prime-age adults are dramatically high in many sub-Saharan African countries, and, as a consequence, the number of orphaned children is high and increasing. In this paper, we identify the educational and health effects of orphanhood in sub-Saharan African countries. We draw on comparable data for eleven sub-Saharan African countries with especially high rates of orphanhood. We define an orphan as a child with at least one deceased biological parent. In the analyzed countries, $10 \%$ or more of children under the age of 15 growing up in private households are orphans (see also Figure 1). In some countries, the rate of orphanhood even exceeds $20 \%{ }^{1}$

It is very likely that the high incidence of parental death not only impacts the demographic composition of the respective countries, but also affects human capital formation. Therefore, on the micro level, we seek to examine how being an orphan affects individual schooling and health outcomes. More specifically, our research compares the educational and health outcomes of orphans living under the same conditions as nonorphans. Differences in outcomes may be interpretable as a product of "Hamilton's Rule," according to which the quality of care depends on the biological connectedness of caregivers and children (Hamilton, 1964). From this perspective, children who grow up without a biological parent are worse off than non-orphaned children growing up under the same living conditions.

Alongside Hamilton's Rule, there are different theories on how the absence of a parent may affect child development (cf. for example Deleire and Kalil, 2002). Economic deprivation in particular may be an immediate consequence of a prime-aged parent's illness and death. As a stylized fact, orphans in sub-Saharan Africa are most likely to live in large households headed by a female adult (cf. for example Monarsch and Boerna, 2004) in which

\footnotetext{
${ }^{1}$ The HIV/AIDS epidemic is one of the primary reasons of deaths among the prime-aged population. In 2009, there were some 14.8 million AIDS orphans in sub-Saharan Africa (UNAIDS, 2010).
} 
multiple household members depend on a single income. Aside from the economic dimension of being an orphan, there are also psychological factors. Previous psychological studies report that AIDS orphans more often suffer from depression or anxiety than other children (cf. for example Atwine et al., 2005). Such mental ailments lead to poorer health and are also likely to have an impact on schooling outcomes. A related problem is stigmatization, which is especially severe for AIDS orphans. In some cases, stigmatized children may even be prevented from attending school. Some of these mechanisms (and especially Hamilton's Rule) are not only relevant for orphans but also for so-called “social orphans.” We define a social orphan as a child whose biological parents are both still alive but who grows up without at least one of his or her biological parents present. In our data, social orphan ratios are higher than orphan ratios (also compare Figure 2). For example, more than half of the observed children in Namibia and Swaziland live without a biological parent. We look separately at the human capital outcomes of orphans and social orphans. ${ }^{2}$ Based on our data, we are also able to observe different family structures, and, in particular, shed light on how the education and health of paternal orphans and paternal social orphans (i.e. children who lack a father) are affected by orphanhood.

The most relevant previous study with respect to our paper is Case et al. (2004), which is based on data from ten sub-Saharan African countries. ${ }^{3}$ Case at al. demonstrate that orphans live in relatively poor households in most of the observed countries. Their main identification strategy is a household fixed-effects strategy that compares orphaned and nonorphaned children within the same household. Based on this fixed-effects estimation, the

\footnotetext{
${ }^{2}$ There are multiple reasons why children are social orphans. First of all, the parents may be separated, with children raised by a single parent. Second, the parents may decide to have their children live with relatives, so that they have better access to education. Children may also be forced to leave their homes in order to work and earn money for their families.

${ }^{3}$ Our literature review is focused on studies that use econometric identification strategies to determine specific effects of orphanhood. We do not summarize the further existing evidence from descriptive studies examining orphans' outcomes in sub-Saharan countries. As an example, Ainsworth and Filmer (2002) provide evidence based on data for 28 developing countries, but do not find clear, systematic evidence of less favorable educational outcomes for orphans.
} 
authors find that orphans and other children not living with a parent tend to be less often enrolled in school than non-orphans. This is especially true for orphans living with less closely related or unrelated caregivers.

A further strand of previous research examines variation over time in order to identify how parental death impacts children's schooling outcomes, thus allowing gradual change in child outcomes to be observed. These studies typically relate to single countries (e.g. Evans and Miguel, 2007 for Kenya; Case and Ardington, 2006 for the KwaZulu-Natal region of South Africa; Ainsworth et al., 2005 for Tanzania). Similarly, Ardington and Leibrandt (2010) use data from South Africa over ten years and examine how the vulnerability of orphans has changed over time as the incidence of parental death has grown due to the AIDS/HIV epidemic. ${ }^{4}$

For us, the previous work by Case et al. (2004) is particularly relevant since it exploits variation within the household using a fixed-effects estimation strategy in order to estimate the causal effect of orphanhood on education. We do not replicate findings concerning household wealth, which are well-documented in the existing literature. However, for the interpretation of our results it is important to bear in mind that living conditions in orphan households are worse than conditions in average non-orphan households. Following Case et al. (2004), we exploit the household fixed-effects strategy using more recent data. ${ }^{5}$

\footnotetext{
${ }^{4}$ Few studies use an instrumental variable approach in order to identify a specific causal effect of orphanhood. For example, Gunderson and Kelly (2008) exploit regional variation in HIV rates in order to instrument for parental death. However, the exogeneity of this instrument may be doubted. Further instruments for adult mortality are discussed in the macro literature linking adult mortality to growth: Lorentzen et al. (2008) use the malaria ecology and different sets of climatic and geographic features as instruments for mortality. We also tested geographical instruments (e.g. administrative district distances to coasts and distances to the equator) in our data, but the correlations between the instruments and orphanhood are very low. Also, one may question what kind of local average treatment effect such instruments would identify. Therefore, we do not rely on an instrumental variable approach based on these instruments in this paper.

${ }^{5}$ This might be crucial since educational policies have changed in many of the considered countries in recent years. Specifically, school fees have been abolished in most countries (with the exception of Swaziland and Zimbabwe), so that primary education is now free of charge (cf. for example World Bank/UNICEF, 2009). In addition, school enrolment rates have increased in recent years (cf. USAID, 2010).
} 
Our work makes several contributions to the existing literature. In contrast to the literature cited above, we do not solely examine the educational outcomes of orphans but also consider nutritional outcomes as an important determinant of child health and human capital. An additional feature of our work is that we distinguish between different family structures. In contrast to Case et al. (2004), we also consider the high numbers of nonorphaned children living without a biological parent and present all of our results for orphans and social orphans.

This paper is organized as follows: In Section 2, we introduce the database and present descriptive insights for the eleven sub-Saharan countries. Section 3 describes our identification strategy and discusses the specific effects we identify. We present the different regression results in Section 4. Section 5 concludes.

In sum, our findings suggest that in most countries, orphans and social orphans growing up under the same conditions as non-orphans are significantly worse off in terms of their observed educational and health outcomes. Compared to children whose parents are alive and live with their biological parents, orphans lag behind in education in all countries. Our estimations indicate that orphaned children lag behind their non-orphan counterparts in cumulative school participation by one-fifth to one-half of a year. Children are especially harmed by not growing up with a biological mother. In most countries, we observe hardly any additional effect of growing up without a father. 


\section{Data and Descriptive Evidence}

This paper is based on comparable data from the Demographic and Health Surveys (DHS) for eleven sub-Saharan African countries. DHS data are collected on behalf of the US Agency for International Development. The data include detailed information on household structure, child health and education. From this database we have chosen those sub-Saharan African countries for which recent data are available and which are known to have high proportions of orphans among children aged 14 and younger (cf. Figure 1). We do not observe institutionalized orphans but only orphans living in households. For this reason, our orphan ratios may be considered to represent a lower bound of the real ratios. Focusing on households is also justified because in the considered countries it is common for orphans to live with their extended families (cf. for example UNICEF, 2006). The specific countries we examine are Kenya, Lesotho, Malawi, Mozambique, Namibia, Rwanda, Swaziland, Tanzania, Uganda, Zambia and Zimbabwe.

The data span from 2003 to 2007 (see Figure 1). Figure 1 shows the ratios of orphans in the observed countries among children under age 15 (i.e. age 14 or younger). ${ }^{6}$ The graph on the right side in Figure 1 focuses on paternal orphans (i.e. children whose biological father is dead). As the graph shows, paternal orphans make up the largest share of all observed orphans. Across all countries, among the $14.8 \%$ of orphans in our sample, $9.2 \%$ are paternal orphans, $2.9 \%$ are maternal orphans and $2.7 \%$ are full orphans. Figure 2 presents the ratios of social orphans among children under age 15 . The percentage of social orphans is even higher (32.0\% averaged over all country averages) than the percentage of orphans (14.8\%).

\footnotetext{
${ }^{6}$ Even though these are unweighted statistics from our data, the ratios are consistent to the numbers published in the respective DHS country reports.
} 
Figure 1: Proportion of orphans among children aged 0-14 in selected sub-Saharan countries

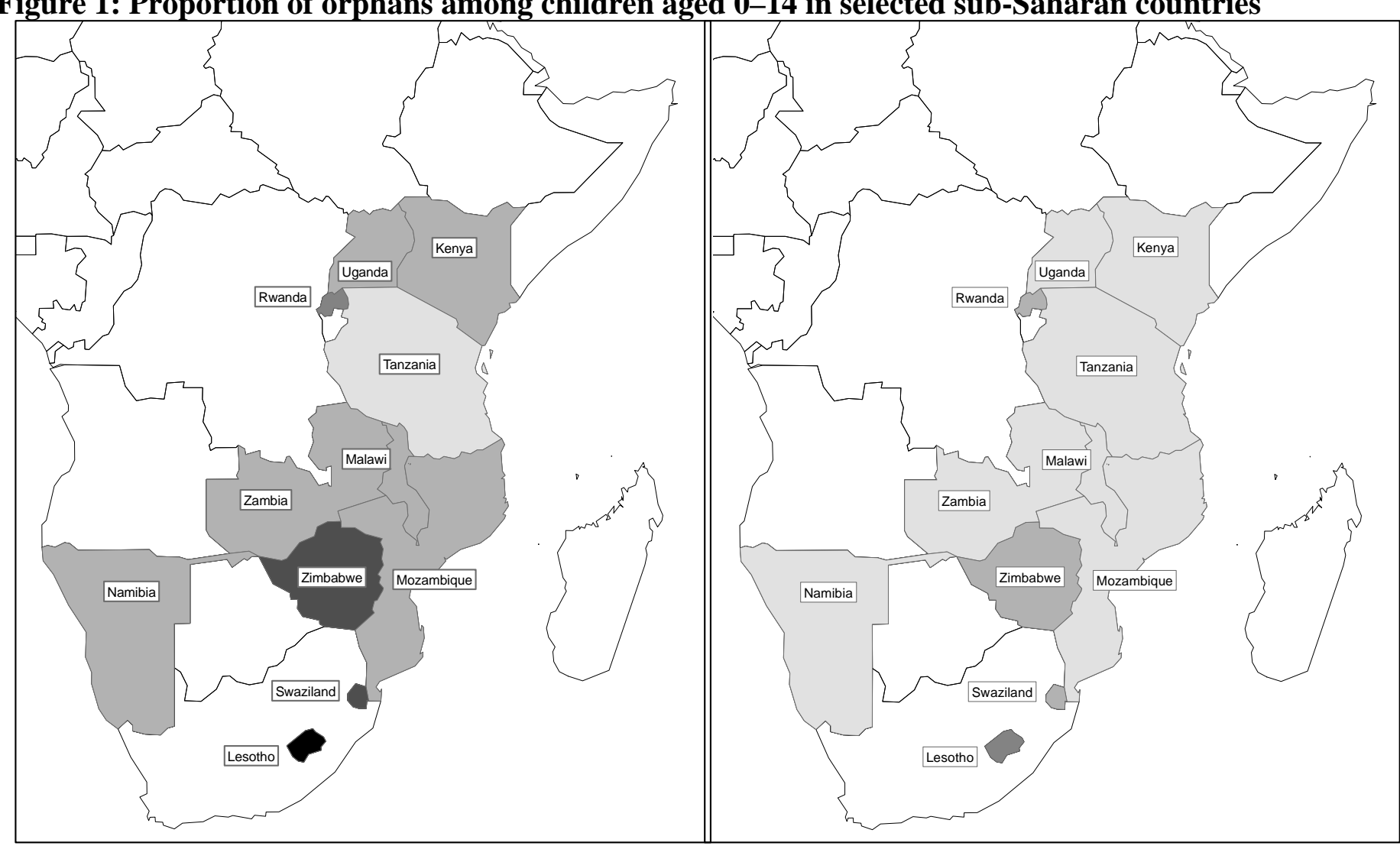

$\square<10,00 \quad 15,00-<20,00$

$15,00-<25,00$

paternal orphan in \%

$\square<10,00$

$15,00-<20,00$

$20,00-<25,00$

Note: Table A 1 in the Appendix presents the numbers underlying this figure.

Source: Demographic and Health Survey (DHS): 2003-2007. Sample of children aged 0-14. Own calculations. 
Figure 2: Proportion of social orphans among children aged 0-14 in selected sub-Saharan countries

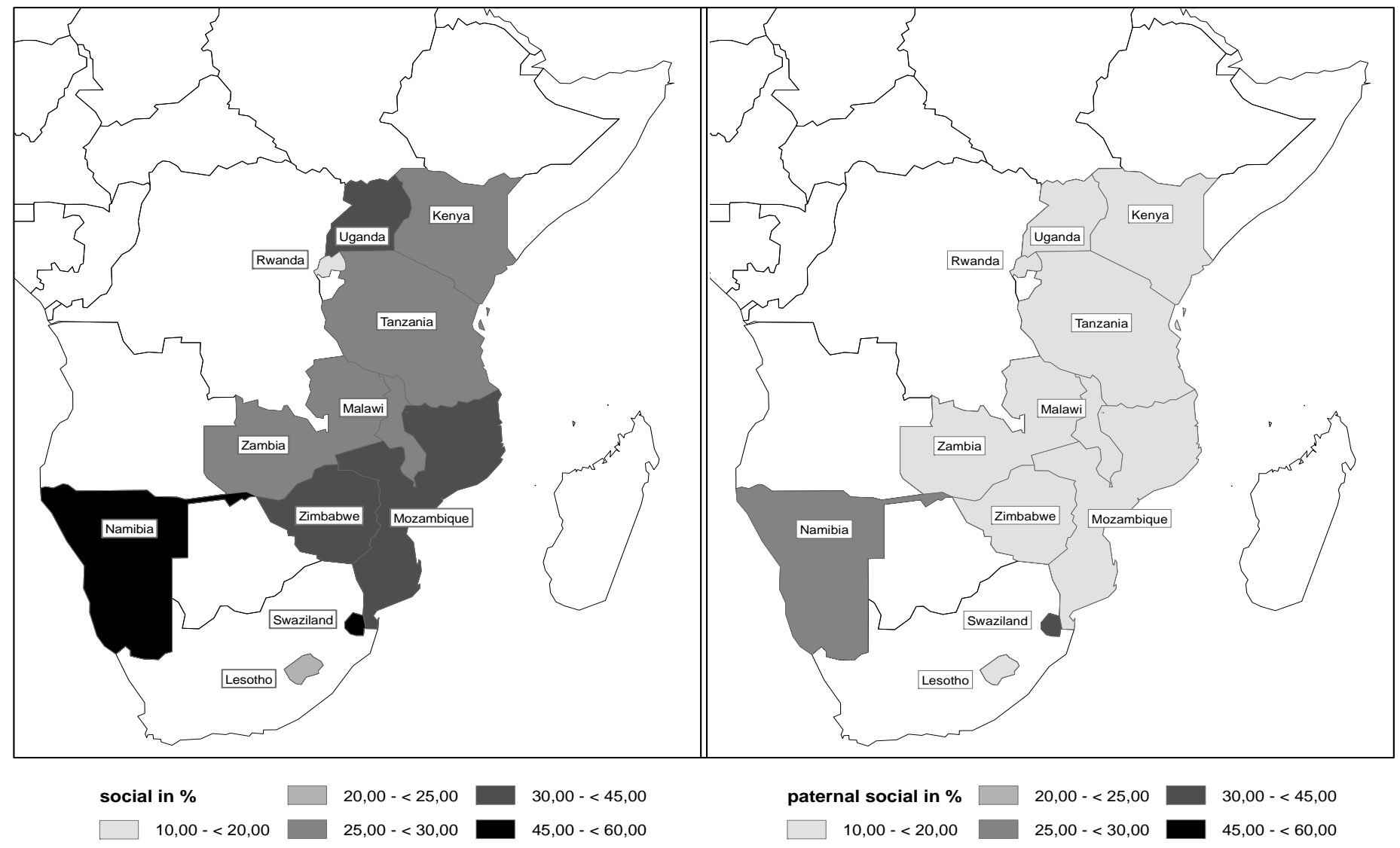

Note: Table A 1 in the Appendix presents the numbers underlying this figure.

Source: Demographic and Health Survey (DHS): 2003-2007. Sample of children aged 0-14. Own calculations. 
For the regression analysis, we construct two sub-samples which we use for our analysis of the orphans' educational and health outcomes, respectively. First, we restrict the country samples to children who are of usual school age. Formal education starts at age six in most countries, with the exception of Rwanda, Tanzania and Zambia, where school starts at age seven (cf. for example Lloyd and Hewett, 2003 for an overview). The oldest children in the school-age sample are 14 years of age. The sample sizes for school-aged children vary between 5,604 (Swaziland) and 16,614 (Malawi). There are few incidences of missing observations for variables needed in the regression analysis. The incidence of missing observations varies between 0 and $4 \%$. We observe all required variables for about $98 \%$ of the children in most countries. The outcome variable we consider is the numbers of years of formal education the children lag behind compared to the years of schooling they should have attained at their observed age (and given the usual age at school entry). According to this measure, lagging behind in education may be the result of a child entering school later than she should have or of an interruption to schooling. ${ }^{7}$

The second sample we look at contains children from birth to age four because we observe various health measures only in this age group. Specifically, we observe standard anthropometric measures like weight-for-age and height-for-age, taking the age and gender of the children into consideration (both measured in z-scores). ${ }^{8}$ Each of these measures implies important health information, and allows us to assess the incidence of "being stunted" and "being underweight." In the DHS data, these measures can be constructed by comparing individual outcomes with the standardized reference distributions provided by the WHO. Accordingly, we define "being stunted" as being below two standard deviations from the

\footnotetext{
${ }^{7}$ We are not able to measure the quality of education the children receive but only observe actual years of education, which our measure compares to the number of years a child should have attended school at the respective age.

${ }^{8}$ These are very precise measures: Weight was measured using lightweight, bathroom-type scales with a digital screen. The height for children younger than 24 months was measured lying down on the board (recumbent length), while standing height was measured for older children.
} 
median height-for-age of the reference population. We classify children whose weight-for-age is below two standard deviations of the reference population as "being underweight.”

Not all children under age five are captured in the country samples. This may have two reasons: First, the surveys had a different focus in each country (and do not all contain the same health measures). Second, not all children could be measured. This may be the case if children (or their parents) refused to be measured or if the child was not immediately present in the household. Thus, depending on the respective country, we observe health measures for some 31 to $91 \%$ of the relevant children. The final sample sizes vary between 1,674 observations for Lesotho and 9,020 observations for Mozambique. Table 1 presents an overview of the different sample sizes relevant for the regression analysis.

Table 1: Sample sizes

\begin{tabular}{lcccccc}
\hline Country, year & \multicolumn{3}{c}{$\begin{array}{c}\text { Education sample } \\
\text { (Age 6/7-14) }\end{array}$} & & \multicolumn{3}{c}{$\begin{array}{c}\text { Health sample } \\
\text { (Age 0-4) }\end{array}$} \\
& $\begin{array}{c}\text { Sample size } \\
\text { without } \\
\text { missing } \\
\text { observations } \\
\text { (absolute) }\end{array}$ & $\begin{array}{c}\text { Sample } \\
\text { without } \\
\text { missing } \\
\text { observations } \\
\text { (\%) }\end{array}$ & $\begin{array}{c}\text { Fixed- } \\
\text { effects } \\
\text { sample* }\end{array}$ & $\begin{array}{c}\text { Sample size } \\
\text { without } \\
\text { missing } \\
\text { observations } \\
\text { (absolute) }\end{array}$ & $\begin{array}{c}\text { Sample } \\
\text { without } \\
\text { missing } \\
\text { observations } \\
\text { (\%) }\end{array}$ & $\begin{array}{c}\text { Fixed- } \\
\text { effects } \\
\text { sample* }\end{array}$ \\
\hline Kenya, 2003 & 9,122 & 96.61 & 7,429 & 5,022 & 85.90 & 2,877 \\
Lesotho, 2004 & 9,055 & 96.00 & 6,781 & 1,674 & 36.71 & 682 \\
Malawi, 2004 & 16,614 & 99.17 & 13,436 & 8,813 & 80.55 & 4,568 \\
Mozambique, 2003 & 16,462 & 98.86 & 13,492 & 9,020 & 84.24 & 5,410 \\
Namibia, 2006-7 & 9,686 & 97.73 & 7,810 & 5,035 & 87.84 & 2,665 \\
Rwanda, 2005 & 10,852 & 100.00 & 7,870 & 3,870 & 47.01 & 2,329 \\
Swaziland, 2006-7 & 5,604 & 97.36 & 4,691 & 2,752 & 88.04 & 1,635 \\
Tanzania, 2004-5 & 11,125 & 99.22 & 8,789 & 7,905 & 91.25 & 4,813 \\
Uganda, 2006 & 13,418 & 99.30 & 11,525 & 2,650 & 30.71 & 1,811 \\
Zambia, 2007 & 8,518 & 99.29 & 6,759 & 5,618 & 87.11 & 3,452 \\
Zimbabwe, 2005-6 & 11,127 & 97.97 & 8,819 & 4,931 & 82.34 & 2,288 \\
\hline \hline
\end{tabular}

Note: *The fixed-effects sample is reduced to children with siblings in the respective age group living in the same household (cf. section 3).

Source: Demographic and Health Survey (DHS): 2003-2007. Own calculations.

Tables 2 to 5 report means and standard deviations for the observed human capital outcomes (education and health outcomes) separately for orphans and non-orphans. In Table 2 we compare the education and health outcomes between orphans and non-orphans in all eleven countries. These summary statistics suggest that - compared to non-orphans - orphans lag behind in education by about half a year in most countries (cf. columns 1 and 2 of Table 2). Additionally, orphans seem to be more likely to suffer from growth retardation, seem to be 
more often stunted and are more likely to be malnourished on average (Table 2). In Table 3 to Table 5, we present summary statistics referring to different groups of orphans.

Table 2: Summary statistics for orphans

\begin{tabular}{|c|c|c|c|c|c|c|c|c|c|c|}
\hline \multirow[b]{2}{*}{$\begin{array}{l}\text { Country } \\
\text { year }\end{array}$} & \multicolumn{2}{|c|}{$\begin{array}{l}\text { years behind } \\
\text { in schooling }\end{array}$} & \multicolumn{2}{|c|}{ height-for-age } & \multicolumn{2}{|c|}{ weight-for-age } & \multicolumn{2}{|c|}{$\begin{array}{l}\text { being } \\
\text { stunted }\end{array}$} & \multicolumn{2}{|c|}{$\begin{array}{c}\text { being } \\
\text { underweight }\end{array}$} \\
\hline & orphan & $\begin{array}{c}\text { other } \\
\text { children }\end{array}$ & orphan & $\begin{array}{c}\text { other } \\
\text { children }\end{array}$ & orphan & $\begin{array}{c}\text { other } \\
\text { children }\end{array}$ & orphan & $\begin{array}{c}\text { other } \\
\text { children }\end{array}$ & orphan & $\begin{array}{c}\text { other } \\
\text { children }\end{array}$ \\
\hline \multirow{2}{*}{$\begin{array}{l}\text { Kenya } \\
2003\end{array}$} & 2.20 & 1.74 & -1.08 & -1.18 & -0.99 & -0.92 & 0.29 & 0.29 & 0.21 & 0.20 \\
\hline & (2.05) & $(2.43)$ & $(1.70)$ & (1.55) & (1.25) & (1.32) & $(0.45)$ & $(0.46)$ & $(0.41)$ & $(0.40)$ \\
\hline \multirow{2}{*}{$\begin{array}{l}\text { Lesotho } \\
2004\end{array}$} & 2.09 & 1.66 & -1.62 & -1.59 & -1.06 & -1.00 & 0.42 & 0.39 & 0.19 & 0.21 \\
\hline & (1.80) & (1.72) & (1.40) & (1.48) & (1.26) & (1.25) & $(0.49)$ & $(0.49)$ & $(0.40)$ & $(0.40)$ \\
\hline \multirow{2}{*}{$\begin{array}{l}\text { Malawi } \\
2004\end{array}$} & 2.09 & 1.78 & -1.97 & -1.84 & -1.17 & -1.07 & 0.51 & 0.48 & 0.24 & 0.22 \\
\hline & $(2.02)$ & (1.94) & (1.79) & (1.62) & (1.30) & (1.23) & $(0.50)$ & $(0.50)$ & $(0.43)$ & $(0.41)$ \\
\hline \multirow{2}{*}{$\begin{array}{l}\text { Mozambique } \\
2003\end{array}$} & 2.93 & 2.39 & -1.67 & -1.65 & -1.10 & -1.09 & 0.41 & 0.39 & 0.19 & 0.22 \\
\hline & (2.92) & $(2.55)$ & $(1.41)$ & (1.43) & $(1.22)$ & (1.23) & $(0.49)$ & $(0.49)$ & $(0.40)$ & $(0.42)$ \\
\hline \multirow{2}{*}{$\begin{array}{l}\text { Namibia } \\
\text { 2006-7 }\end{array}$} & 1.36 & 1.05 & -1.28 & -1.06 & -1.37 & -1.02 & 0.27 & 0.24 & 0.28 & 0.22 \\
\hline & (1.65) & (1.58) & (1.49) & $(1.42)$ & (1.24) & $(1.25)$ & $(0.45)$ & $(0.43)$ & $(0.45)$ & $(0.41)$ \\
\hline \multirow{2}{*}{$\begin{array}{l}\text { Rwanda } \\
2005\end{array}$} & 2.47 & 1.84 & -1.87 & -1.74 & -1.26 & -1.09 & 0.49 & 0.45 & 0.20 & 0.22 \\
\hline & (1.84) & (2.07) & $(1.50)$ & $(1.52)$ & (1.05) & (1.23) & $(0.50)$ & $(0.50)$ & $(0.40)$ & $(0.42)$ \\
\hline \multirow{2}{*}{$\begin{array}{l}\text { Swaziland } \\
2006-7\end{array}$} & 1.70 & 1.27 & -1.25 & -1.07 & -0.58 & -0.41 & 0.32 & 0.23 & 0.11 & 0.07 \\
\hline & (1.70) & (1.57) & (1.49) & (1.37) & (1.43) & (1.21) & $(0.47)$ & $(0.42)$ & $(0.32)$ & $(0.25)$ \\
\hline \multirow{2}{*}{$\begin{array}{l}\text { Tanzania } \\
\text { 2004-5 }\end{array}$} & 1.88 & 1.43 & -1.83 & -1.66 & -1.29 & -1.15 & 0.47 & 0.39 & 0.29 & 0.23 \\
\hline & (1.82) & (4.17) & (1.65) & (1.28) & (1.17) & (1.13) & $(0.50)$ & $(0.49)$ & $(0.45)$ & $(0.42)$ \\
\hline \multirow{2}{*}{$\begin{array}{l}\text { Uganda } \\
2006\end{array}$} & 2.14 & 1.74 & -1.46 & -1.38 & -1.09 & -1.04 & 0.29 & 0.33 & 0.22 & 0.21 \\
\hline & (3.77) & $(4.92)$ & (1.58) & (1.50) & (1.22) & (1.25) & $(0.46)$ & $(0.47)$ & $(0.42)$ & $(0.41)$ \\
\hline \multirow{2}{*}{$\begin{array}{l}\text { Zambia } \\
2007\end{array}$} & 1.09 & 0.95 & -1.52 & -1.48 & -1.18 & -0.96 & 0.43 & 0.38 & 0.25 & 0.19 \\
\hline & (1.75) & (1.64) & (1.66) & (1.60) & (1.14) & (1.21) & $(0.50)$ & $(0.49)$ & $(0.44)$ & (0.39) \\
\hline \multirow{2}{*}{$\begin{array}{l}\text { Zimbabwe } \\
\text { 2005-6 }\end{array}$} & 0.36 & 0.24 & -1.47 & -1.21 & -1.13 & -0.83 & 0.38 & 0.28 & 0.22 & 0.16 \\
\hline & (7.41) & (6.97) & (1.59) & (1.54) & (1.19) & (1.27) & $(0.48)$ & $(0.45)$ & $(0.42)$ & $(0.36)$ \\
\hline
\end{tabular}

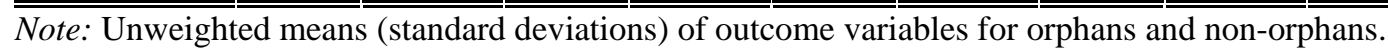

Source: Demographic and Health Survey (DHS): 2003-2007. Means and (standard deviations). Educational outcomes refer to the samples of children aged 6/7-14. Health outcomes are based on the samples of children aged $0-4$. The respective sample sizes are shown in Table 1. Own calculations.

Specifically, we distinguish between paternal orphans and orphans whose mother has died (maternal orphans as well as full orphans). We do not distinguish between maternal and full orphans since the proportion of each group is relatively low compared to the proportion of paternal orphans (cf. Figure 1). A comparison of columns 1 and 2 in Table 3 suggests that on average, maternal orphans and full orphans lag more years behind in education than paternal orphans. However, the mean difference in years of education is less than half a year. For our health outcomes, children are somewhat more often stunted and more likely to be underweight in the case of paternal death. This result could be driven by financial constraints 
caused by the death of the father. However, for most countries the mean differences between paternal and non-paternal orphans are rather small and statistically insignificant.

Table 3: Summary statistics for paternal orphans

\begin{tabular}{|c|c|c|c|c|c|c|c|c|c|c|}
\hline \multirow[b]{2}{*}{$\begin{array}{l}\text { Country, } \\
\text { year }\end{array}$} & \multicolumn{2}{|c|}{$\begin{array}{l}\text { years behind } \\
\text { in schooling }\end{array}$} & \multicolumn{2}{|c|}{ height-for-age } & \multicolumn{2}{|c|}{ weight-for-age } & \multicolumn{2}{|c|}{$\begin{array}{c}\text { being } \\
\text { stunted }\end{array}$} & \multicolumn{2}{|c|}{$\begin{array}{c}\text { being } \\
\text { underweight }\end{array}$} \\
\hline & $\begin{array}{c}\text { paternal } \\
\text { orphan }\end{array}$ & $\begin{array}{c}\text { other } \\
\text { children }\end{array}$ & $\begin{array}{c}\text { paternal } \\
\text { orphan }\end{array}$ & $\begin{array}{c}\text { other } \\
\text { children }\end{array}$ & $\begin{array}{c}\text { paternal } \\
\text { orphan }\end{array}$ & $\begin{array}{c}\text { other } \\
\text { children }\end{array}$ & $\begin{array}{c}\text { paternal } \\
\text { orphan }\end{array}$ & $\begin{array}{c}\text { other } \\
\text { children }\end{array}$ & $\begin{array}{c}\text { paternal } \\
\text { orphan }\end{array}$ & $\begin{array}{l}\text { other } \\
\text { children }\end{array}$ \\
\hline \multirow{2}{*}{$\begin{array}{l}\text { Kenya } \\
2003\end{array}$} & 2.18 & 2.22 & -1.18 & -1.18 & -0.98 & -0.92 & 0.29 & 0.29 & 0.19 & 0.20 \\
\hline & (2.03) & $(2.08)$ & $(1.58)$ & $(1.56)$ & $(1.20)$ & (1.32) & $(0.46)$ & $(0.46)$ & $(0.40)$ & $(0.40)$ \\
\hline \multirow{2}{*}{$\begin{array}{l}\text { Lesotho } \\
2004\end{array}$} & 2.00 & 2.27 & -1.63 & -1.59 & -1.05 & -1.00 & 0.42 & 0.39 & 0.19 & 0.21 \\
\hline & (1.81) & (1.75) & (1.34) & (1.49) & (1.27) & (1.25) & $(0.50)$ & $(0.49)$ & $(0.40)$ & $(0.40)$ \\
\hline \multirow{2}{*}{$\begin{array}{l}\text { Malawi } \\
2004\end{array}$} & 2.02 & 2.20 & -1.99 & -1.84 & -1.14 & -1.07 & 0.50 & 0.48 & 0.21 & 0.22 \\
\hline & $(2.02)$ & $(2.02)$ & $(17.3)$ & $(1.62)$ & (1.28) & (1.23) & $(0.50)$ & $(0.50)$ & $(0.41)$ & $(0.41)$ \\
\hline \multirow{2}{*}{$\begin{array}{l}\text { Mozambique } \\
2003\end{array}$} & 2.87 & 3.02 & -1.72 & -1.65 & -1.13 & -1.09 & 0.41 & 0.39 & 0.19 & 0.22 \\
\hline & (2.18) & (3.84) & $(1.31)$ & (1.43) & (1.17) & (1.23) & $(0.49)$ & $(0.49)$ & (0.39) & $(0.41)$ \\
\hline \multirow{2}{*}{$\begin{array}{l}\text { Namibia } \\
2006-7\end{array}$} & 1.23 & 1.55 & -1.19 & -1.07 & -1.30 & -1.03 & 0.24 & 0.25 & 0.28 & 0.22 \\
\hline & (1.52) & (1.81) & (1.44) & $(1.42)$ & (1.28) & (1.25) & $(0.43)$ & $(0.43)$ & $(0.45)$ & $(0.41)$ \\
\hline \multirow{2}{*}{$\begin{array}{l}\text { Rwanda } \\
2005\end{array}$} & 2.35 & 2.71 & -1.76 & -1.74 & -1.26 & -1.09 & 0.46 & 0.45 & 0.20 & 0.22 \\
\hline & $(1.80)$ & (1.89) & $(1.48)$ & $(1.52)$ & (1.06) & (1.23) & $(0.50)$ & $(0.50)$ & $(0.40)$ & $(0.42)$ \\
\hline \multirow{2}{*}{$\begin{array}{l}\text { Swaziland } \\
\text { 2006-7 }\end{array}$} & 1.61 & 1.84 & -1.30 & -1.07 & -0.53 & -0.42 & 0.31 & 0.24 & 0.05 & 0.07 \\
\hline & (1.66) & (1.75) & $(1.29)$ & (1.38) & $(1.22)$ & (1.23) & $(0.47)$ & $(0.42)$ & $(0.23)$ & $(0.26)$ \\
\hline \multirow{2}{*}{$\begin{array}{l}\text { Tanzania } \\
2004-5\end{array}$} & 1.81 & 1.98 & -1.67 & -1.67 & -1.23 & -1.16 & 0.43 & 0.40 & 0.27 & 0.23 \\
\hline & (1.81) & (1.83) & $(1.60)$ & (1.28) & (1.14) & (1.14) & $(0.50)$ & $(0.49)$ & $(0.44)$ & $(0.42)$ \\
\hline \multirow{2}{*}{$\begin{array}{l}\text { Uganda } \\
2006\end{array}$} & 2.19 & 2.07 & -1.42 & -1.38 & -1.08 & -1.04 & 0.25 & 0.33 & 0.21 & 0.21 \\
\hline & (3.09) & $(4.50)$ & $(1.45)$ & (1.51) & (1.17) & $(1.25)$ & $(0.44)$ & $(0.47)$ & $(0.41)$ & $(0.41)$ \\
\hline \multirow{2}{*}{$\begin{array}{l}\text { Zambia } \\
2007\end{array}$} & 0.97 & 1.23 & -1.38 & -1.49 & -1.20 & -0.97 & 0.40 & 0.39 & 0.25 & 0.19 \\
\hline & (1.65) & (1.85) & (1.64) & (1.60) & (1.17) & (1.21) & $(0.49)$ & $(0.49)$ & $(0.44)$ & (0.39) \\
\hline \multirow{2}{*}{$\begin{array}{l}\text { Zimbabwe } \\
\text { 2005-6 }\end{array}$} & 0.50 & 0.17 & -1.48 & -1.21 & -1.07 & -0.84 & 0.37 & 0.29 & 0.19 & 0.16 \\
\hline & (5.95) & $(9.06)$ & $(1.45)$ & $(1.55)$ & $(1.15)$ & $(1.27)$ & $(0.48)$ & $(0.45)$ & $(0.40)$ & $(0.37)$ \\
\hline
\end{tabular}

Note: Unweighted means (standard deviations) of outcome variables for paternal orphans (p-orphan) in comparison to all other children (non-p-orphan).

Source: Demographic and Health Survey (DHS): 2003-2007. Means and (standard deviations). Educational outcomes refer to the samples of children aged 6/7-14. Health outcomes are based on the samples of children aged 0-4. Own calculations.

Table 4 shows summary statistics of the outcomes for social orphans. The differences between social orphans and non-social orphans are mostly small and statistically insignificant. This result might reflect the fact that there are multiple reasons for living without a biological parent which differ across countries. In Table 5, we distinguish between social orphans not living with the father (but with a mother) and all other social orphans (i.e. those not living with a mother). Regarding educational outcomes, paternal social orphans mostly seem to lag somewhat behind in education. Again, the health outcomes are very similar for both groups. 
Table 4: Summary statistics for social orphans

\begin{tabular}{|c|c|c|c|c|c|c|c|c|c|c|}
\hline \multirow[b]{2}{*}{$\begin{array}{l}\text { Country } \\
\text { year }\end{array}$} & \multicolumn{2}{|c|}{$\begin{array}{l}\text { years behind } \\
\text { in schooling }\end{array}$} & \multicolumn{2}{|c|}{ height-for age } & \multicolumn{2}{|c|}{ weight-for-age } & \multicolumn{2}{|c|}{$\begin{array}{l}\text { being } \\
\text { stunted }\end{array}$} & \multicolumn{2}{|c|}{$\begin{array}{c}\text { being } \\
\text { underweight }\end{array}$} \\
\hline & $\begin{array}{c}\text { social } \\
\text { orphan }\end{array}$ & $\begin{array}{c}\text { other } \\
\text { children }\end{array}$ & $\begin{array}{c}\text { social } \\
\text { orphan }\end{array}$ & $\begin{array}{c}\text { other } \\
\text { children }\end{array}$ & $\begin{array}{c}\text { social } \\
\text { orphan }\end{array}$ & $\begin{array}{c}\text { other } \\
\text { children }\end{array}$ & $\begin{array}{c}\text { social } \\
\text { orphan }\end{array}$ & $\begin{array}{c}\text { other } \\
\text { children }\end{array}$ & $\begin{array}{c}\text { social } \\
\text { orphan }\end{array}$ & $\begin{array}{c}\text { other } \\
\text { children }\end{array}$ \\
\hline \multirow{2}{*}{$\begin{array}{l}\text { Kenya } \\
2003\end{array}$} & 1.80 & 1.82 & -1.17 & -1.18 & -0.88 & -0.94 & 0.28 & 0.30 & 0.20 & 0.20 \\
\hline & (3.21) & (1.92) & (1.60) & (1.55) & (1.35) & (1.30) & $(0.45)$ & $(0.46)$ & $(0.40)$ & $(0.40)$ \\
\hline \multirow{2}{*}{$\begin{array}{l}\text { Lesotho } \\
2004\end{array}$} & 1.77 & 1.81 & -1.49 & -1.64 & -0.89 & -1.05 & 0.37 & 0.40 & 0.18 & 0.21 \\
\hline & (1.85) & (1.73) & (1.56) & $(1.42)$ & (1.29) & (1.23) & $(0.48)$ & $(0.49)$ & $(0.39)$ & $(0.41)$ \\
\hline \multirow{2}{*}{$\begin{array}{l}\text { Malawi } \\
2004\end{array}$} & 1.90 & 1.82 & -1.92 & -1.82 & -1.08 & -1.07 & 0.49 & 0.48 & 0.22 & 0.22 \\
\hline & (1.98) & (1.95) & (1.64) & (1.63) & $(1.21)$ & (1.24) & $(0.50)$ & $(0.50)$ & $(0.41)$ & $(0.41)$ \\
\hline \multirow{2}{*}{$\begin{array}{l}\text { Mozambique } \\
2003\end{array}$} & 2.47 & 2.48 & -1.61 & -1.67 & -1.04 & -1.10 & 0.38 & 0.40 & 0.20 & 0.23 \\
\hline & $(2.82)$ & (2.51) & $(1.42)$ & (1.43) & $(1.25)$ & (1.23) & $(0.48)$ & $(0.49)$ & $(0.40)$ & $(0.42)$ \\
\hline \multirow{2}{*}{$\begin{array}{l}\text { Namibia } \\
2006-7\end{array}$} & 1.09 & 1.15 & -1.06 & -1.10 & -1.06 & -1.00 & 0.24 & 0.25 & 0.22 & 0.21 \\
\hline & (1.57) & (1.63) & (1.41) & (1.44) & $(1.21)$ & (1.32) & $(0.43)$ & $(0.43)$ & $(0.42)$ & $(0.41)$ \\
\hline \multirow{2}{*}{$\begin{array}{l}\text { Rwanda } \\
2005\end{array}$} & 2.04 & 2.02 & -1.74 & -1.74 & -1.15 & -1.08 & 0.43 & 0.45 & 0.25 & 0.21 \\
\hline & (1.80) & (2.08) & (1.52) & (1.52) & (1.24) & $(1.22)$ & $(0.50)$ & $(0.50)$ & $(0.43)$ & $(0.41)$ \\
\hline \multirow{2}{*}{$\begin{array}{l}\text { Swaziland } \\
\text { 2006-7 }\end{array}$} & 1.30 & 1.48 & -1.11 & -1.03 & -0.45 & -0.38 & 0.24 & 0.23 & 0.07 & 0.07 \\
\hline & (1.54) & (1.69) & (1.37) & (1.40) & (1.19) & (1.31) & $(0.43)$ & $(0.42)$ & $(0.26)$ & $(0.25)$ \\
\hline \multirow{2}{*}{$\begin{array}{l}\text { Tanzania } \\
\text { 2004-5 }\end{array}$} & 1.45 & 1.52 & -1.72 & -1.65 & -1.20 & -1.14 & 0.42 & 0.39 & 0.24 & 0.23 \\
\hline & (5.23) & (3.13) & (1.31) & (1.29) & (1.14) & (1.13) & (0.49) & (0.49) & $(0.43)$ & $(0.42)$ \\
\hline \multirow{2}{*}{$\begin{array}{l}\text { Uganda } \\
2006\end{array}$} & 1.77 & 1.84 & -1.36 & -1.39 & -1.06 & -1.04 & 0.35 & 0.33 & 0.22 & 0.21 \\
\hline & (5.14) & (4.50) & (1.56) & (1.48) & (1.27) & (1.24) & (0.48) & $(0.47)$ & (0.41) & $(0.41)$ \\
\hline \multirow{2}{*}{$\begin{array}{l}\text { Zambia } \\
2007\end{array}$} & 1.05 & 0.95 & -1.42 & -1.51 & -0.99 & -0.96 & 0.38 & 0.39 & 0.20 & 0.19 \\
\hline & (1.69) & (1.65) & (1.61) & (1.60) & (1.19) & (1.21) & $(0.49)$ & $(0.49)$ & $(0.40)$ & $(0.39)$ \\
\hline \multirow{2}{*}{$\begin{array}{l}\text { Zimbabwe } \\
\text { 2005-6 }\end{array}$} & 0.19 & 0.33 & -1.24 & -1.22 & -0.85 & -0.85 & 0.29 & 0.29 & 0.16 & 0.16 \\
\hline & $(7.26)$ & $(7.02)$ & (1.56) & (1.53) & (1.28) & $(1.26)$ & $(0.46)$ & $(0.45)$ & $(0.37)$ & $(0.37)$ \\
\hline
\end{tabular}

Note: Unweighted means (standard deviations) of outcome variables for social orphans (social) in comparison to all other children (non-social).

Source: Demographic and Health Survey (DHS): 2003-2007. Means and (standard deviations). Educational outcomes refer to the samples of children aged 6/7-14. Health outcomes are based on the samples of children aged 0-4. Own calculations.

However, a comparison of means hardly allows conclusions to be drawn concerning whether orphans perform better or worse than non-orphans. The different groups are not directly comparable. For example, Case et al. (2004) demonstrate that orphans live in poorer households and under worse conditions than non-orphans. Also, orphans are on average older than non-orphans. In our data, they are 3.57 years older on average, while the largest mean age difference between orphans and non-orphans is 4.62 years in Rwanda. A simple comparison of means conceals such differences. 
Table 5: Summary statistics for paternal social orphans

\begin{tabular}{|c|c|c|c|c|c|c|c|c|c|c|}
\hline \multirow[b]{2}{*}{$\begin{array}{l}\text { Country } \\
\text { year }\end{array}$} & \multicolumn{2}{|c|}{$\begin{array}{l}\text { years behind } \\
\text { in schooling }\end{array}$} & \multicolumn{2}{|c|}{ height-for age } & \multicolumn{2}{|c|}{ weight-for-age } & \multicolumn{2}{|c|}{$\begin{array}{l}\text { being } \\
\text { stunted }\end{array}$} & \multicolumn{2}{|c|}{$\begin{array}{c}\text { being } \\
\text { underweight }\end{array}$} \\
\hline & $\begin{array}{c}\text { paternal } \\
\text { social } \\
\text { orphan }\end{array}$ & $\begin{array}{c}\text { other } \\
\text { children }\end{array}$ & $\begin{array}{c}\text { paternal } \\
\text { social } \\
\text { orphan }\end{array}$ & $\begin{array}{l}\text { other } \\
\text { children }\end{array}$ & $\begin{array}{c}\text { paternal } \\
\text { social } \\
\text { orphan }\end{array}$ & $\begin{array}{l}\text { other } \\
\text { children }\end{array}$ & $\begin{array}{c}\text { paternal } \\
\text { social } \\
\text { orphan }\end{array}$ & $\begin{array}{l}\text { other } \\
\text { children }\end{array}$ & $\begin{array}{c}\text { paternal } \\
\text { social } \\
\text { orphan }\end{array}$ & $\begin{array}{c}\text { other } \\
\text { children }\end{array}$ \\
\hline Kenya & 1.55 & 2.14 & -1.18 & -1.17 & -0.87 & -0.94 & 0.28 & 0.30 & 0.19 & 0.20 \\
\hline 2003 & (3.00) & (3.46) & (1.57) & (1.56) & (1.35) & (1.30) & $(0.45)$ & $(0.46)$ & $(0.40)$ & $(0.40)$ \\
\hline Lesotho & 1.41 & 1.91 & -1.42 & -1.64 & -0.82 & -1.05 & 0.33 & 0.41 & 0.17 & 0.21 \\
\hline 2004 & (1.66) & (1.90) & (1.46) & (1.47) & (1.31) & (1.23) & $(0.47)$ & $(0.49)$ & $(0.37)$ & $(0.41)$ \\
\hline Malawi & 1.94 & 1.87 & -1.87 & -1.84 & -1.08 & -1.07 & 0.48 & 0.48 & 0.22 & 0.21 \\
\hline 2004 & (1.97) & (1.98) & (1.64) & (1.63) & (1.24) & (1.23) & $(0.50)$ & $(0.50)$ & $(0.42)$ & $(0.41)$ \\
\hline Mozambique & 2.26 & 2.66 & -1.59 & -1.67 & -1.04 & -1.10 & 0.36 & 0.40 & 0.21 & 0.22 \\
\hline 2003 & (2.76) & $(2.86)$ & (1.39) & (1.44) & (1.27) & $(1.22)$ & $(0.48)$ & $(0.49)$ & $(0.41)$ & $(0.42)$ \\
\hline Namibia & 1.00 & 1.16 & -1.07 & -1.07 & -1.03 & -1.04 & 0.24 & 0.25 & 0.22 & 0.22 \\
\hline 2006-7 & (1.49) & (1.61) & (1.36) & (1.47) & (1.22) & (1.27) & $(0.43)$ & $(0.43)$ & $(0.42)$ & $(0.41)$ \\
\hline Rwanda & 1.98 & 2.11 & -1.71 & -1.75 & -1.17 & -1.08 & 0.43 & 0.45 & 0.26 & 0.21 \\
\hline 2005 & (1.71) & (1.89) & (1.55) & (1.51) & (1.25) & (1.21) & $(0.49)$ & $(0.50)$ & $(0.44)$ & $(0.41)$ \\
\hline Swaziland & 1.13 & 1.46 & -1.04 & -1.12 & -0.41 & -0.44 & 0.22 & 0.26 & 0.07 & 0.07 \\
\hline 2006-7 & (1.44) & (1.61) & (1.31) & (1.43) & (1.19) & $(1.26)$ & $(0.41)$ & $(0.44)$ & $(0.25)$ & $(0.26)$ \\
\hline Tanzania & 1.38 & 1.50 & -1.70 & -1.66 & -1.18 & -1.15 & 0.41 & 0.39 & 0.24 & 0.23 \\
\hline 2004-5 & (5.24) & (5.23) & (1.27) & (1.30) & (1.62) & (1.13) & (0.49) & $(0.49)$ & $(0.43)$ & $(0.42)$ \\
\hline Uganda & 1.72 & 1.81 & -1.36 & -1.38 & -1.10 & -1.03 & 0.34 & 0.33 & 0.22 & 0.21 \\
\hline 2006 & (5.61) & $(4.82)$ & (1.50) & (1.51) & (1.25) & $(1.25)$ & $(0.47)$ & $(0.47)$ & $(0.42)$ & $(0.41)$ \\
\hline Zambia & 0.97 & 1.10 & -1.45 & -1.50 & -1.02 & -0.96 & 0.38 & 0.39 & 0.21 & 0.19 \\
\hline 2007 & (1.60) & (1.74) & (1.62) & (1.60) & (1.20) & (1.21) & $(0.49)$ & $(0.49)$ & $(0.40)$ & (0.39) \\
\hline Zimbabwe & 0.19 & 0.19 & -1.27 & -1.21 & -0.84 & -0.86 & 0.29 & 0.29 & 0.16 & 0.17 \\
\hline 2005-6 & (6.56) & (7.78) & $(1.47)$ & (1.57) & (1.30) & $(1.26)$ & $(0.45)$ & $(0.45)$ & $(0.36)$ & $(0.37)$ \\
\hline
\end{tabular}

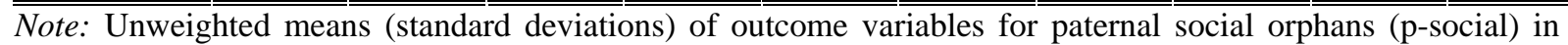
comparison to all other children (non-p-social).

Source: Demographic and Health Survey (DHS): 2003-2007. Means and (standard deviations). Educational outcomes refer to the samples of children aged 6/7-14. Health outcomes are based on the samples of children aged $0-4$. Own calculations.

\section{Identification Strategy}

A major limitation of many of the previous descriptive studies is that they do not consider the “endogeneity” of being an orphan: The observed relationships between orphanhood and child outcomes could reflect unobserved factors correlated with both being an orphan and human capital. For example, wealthier parents might be more educated and more likely to invest in their health (including protection against HIV). At the same time these families are also more likely to invest in their children's human capital in terms of schooling and nutrition. If we do not take these factors into account, any estimate of the effect of being an orphan on child 
outcomes will be biased. Specifically, the effects of lagging behind in education and suffering from negative health outcomes would probably be overestimated.

Our identification strategy is based on a household fixed-effects model. Using a household fixed-effect approach allows us to compare orphans and non-orphans living under similar conditions (i.e. in the same household). Note that living in an extended family is the usual family context in the countries of interest (cf. Section 2). In the case of parental death, the extended family usually cares for orphaned children. Consequently, it is very common for orphaned and non-orphaned children to live together in the same household (i.e. in "blended families”). The average family size in our fixed-effects sample is seven to eight household members in all countries. Only about $4 \%$ of the observed children do not live with a related household head. For orphans, the proportion of children not living with related household heads varies between $4.47 \%$ (Uganda) and 12.59\% (Zambia). This demonstrates that mostly the extended family cares for orphaned children. The numbers are similar in the health sample: In this sample with younger children, the average family size varies between six or seven household members in all countries. Averaged across countries, only about $2 \%$ of the very young children do not live with a related household head. The percentage of orphans who are cared for by non-related persons varies between and $0.47 \%$ in Lesotho and $12.92 \%$ in Zambia.

Specifically, in our fixed-effects estimation, we regress our outcomes of interest on the indicator variables for orphan status and control variables (gender, age and age squared), including household fixed effects. ${ }^{9}$ For the education sample, we show results for three specifications: In specification 1, we solely include an orphan indicator (besides the control variables). In specification 2, we additionally include an indicator for being a social orphan. In specification 3, we include two more indicator variables for being a paternal orphan and a

\footnotetext{
${ }^{9}$ We control for age since non-orphans are often younger than orphans (cf. Section 3). We also tried higher order polynomial specifications of age and age dummies which did not change the presented results. We do not control for age when considering the age adjusted health outcomes. For the binary health outcomes "being stunted" and "being underweight," we estimate linear probability models.
} 
paternal social orphan, respectively. Because of the lower number of observations, we only use the first and second specification when examining health outcomes. ${ }^{10}$

What effect is identified based on the household fixed-effects strategy? This estimation method allows us to control for (economic) living conditions. In other words, we can deduce whether orphans are worse off than non-orphans even when they are not currently experiencing worse (economic) circumstances. If we find differences in education and health outcomes between orphans and non-orphans, this may be an effect of Hamilton's Rule. However, it is very likely that orphans experienced lower parental investments prior to living in the blended households. For example, if parents suffer from an illness several years before they die, they may be less able to earn money and invest in their children. Therefore, the effect we measure is not a direct measure of Hamilton's Rule but a joint effect of the investment they received prior to and after parental death. This effect is still of relevance for social and aid policy. Policymakers are interested in identifying those children most in need of educational and health support. If we identify that orphans (including social orphans) lag behind non-orphans living under the same conditions, this will highlight the need to provide targeted assistance to these children.

We also conduct a robustness check in order to see whether the thus estimated "orphan effect” declines after the orphans have lived in blended households for several years. Since we do not have data on the time of parental death, we restrict our education sample to children living in blended households where there are no orphans younger than age six. We assume that parents have children up to when they begin to suffer severely from a fatal illness or up to death. Thus, if there are no orphans younger than six years of age, the parents probably died several years ago. If the orphan effect is robust for these orphans without any "recent" siblings, this indicates that the estimated effects on human capital are stable over time.

\footnotetext{
${ }^{10}$ There are fewer orphans aged four and younger: In our health sample, $5.27 \%$ of these young children are orphans. The incidence of being a social orphan is about $34.46 \%$ in this group.
} 


\section{Regression Results}

Figure 3 provides insight into the question of whether orphans lag behind in education compared to non-orphaned children growing up under identical living conditions. The point estimates of the orphan effect obtained from the fixed-effects regressions in our first specification are positive for all eleven sub-Saharan countries. Also, the effects are statistically significant for most countries with the exception of Namibia and Uganda. The point estimates are highest in Kenya, Lesotho and Rwanda (and equal about one-third of a year of education, cf. also Table A 2 in the Appendix).

\section{Figure 3: Fixed-effects estimates (specification 1): Educational outcomes}

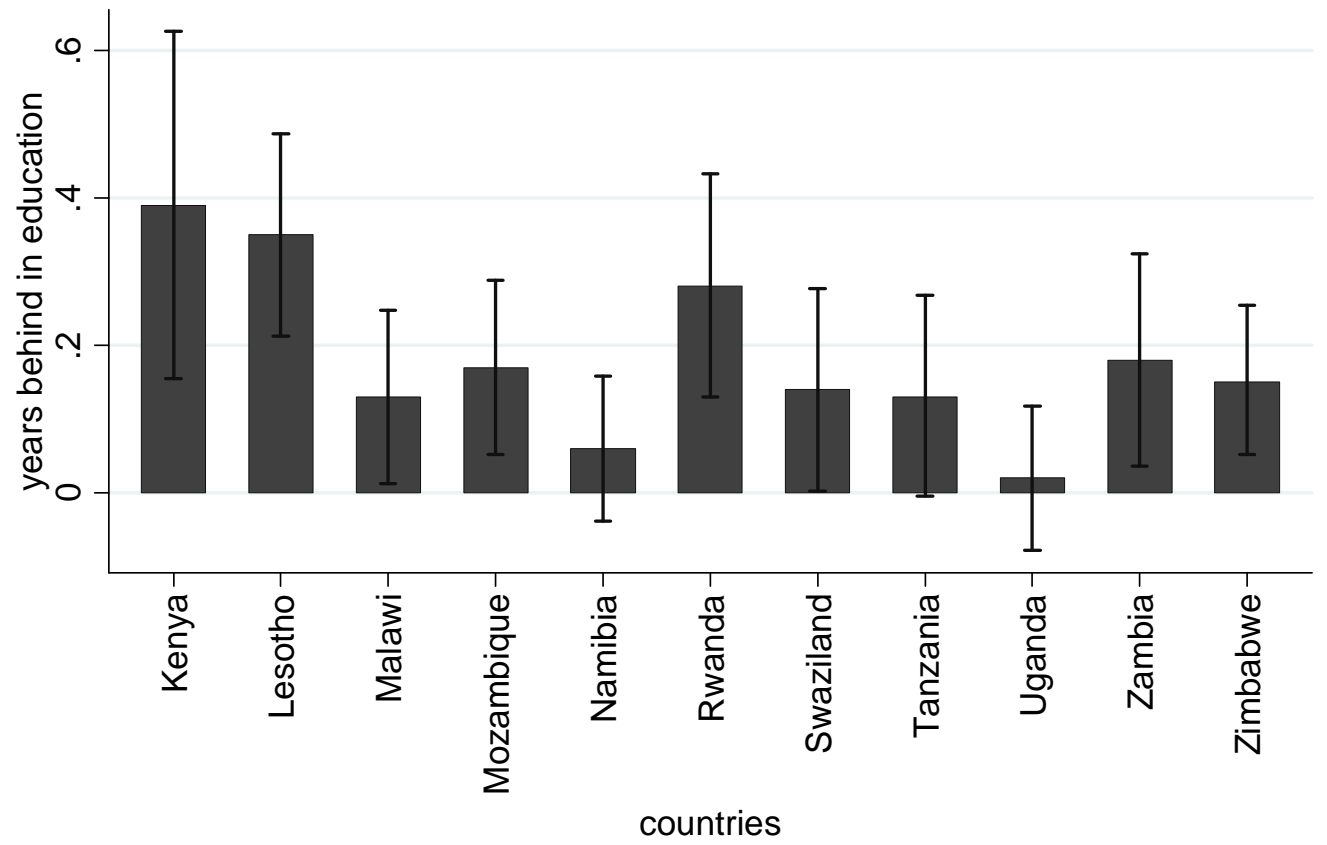

Note: Household fixed-effects regressions include control variables for age, age squared and gender (bars = coefficients, lines $=95 \%$ confidence bounds). Estimated separately by country. Specification 1 refers to a binary variable indicating orphanhood (reference category: at least one parent is alive). The point estimates underlying Figure 3 are presented in Table A 2 in the Appendix.

Source: Demographic and Health Survey (DHS): 2003-2007. Sample restricted to children aged 6/7-14. Own estimations. 
Figure 4: Fixed-effects estimates (specification 2): Educational outcomes

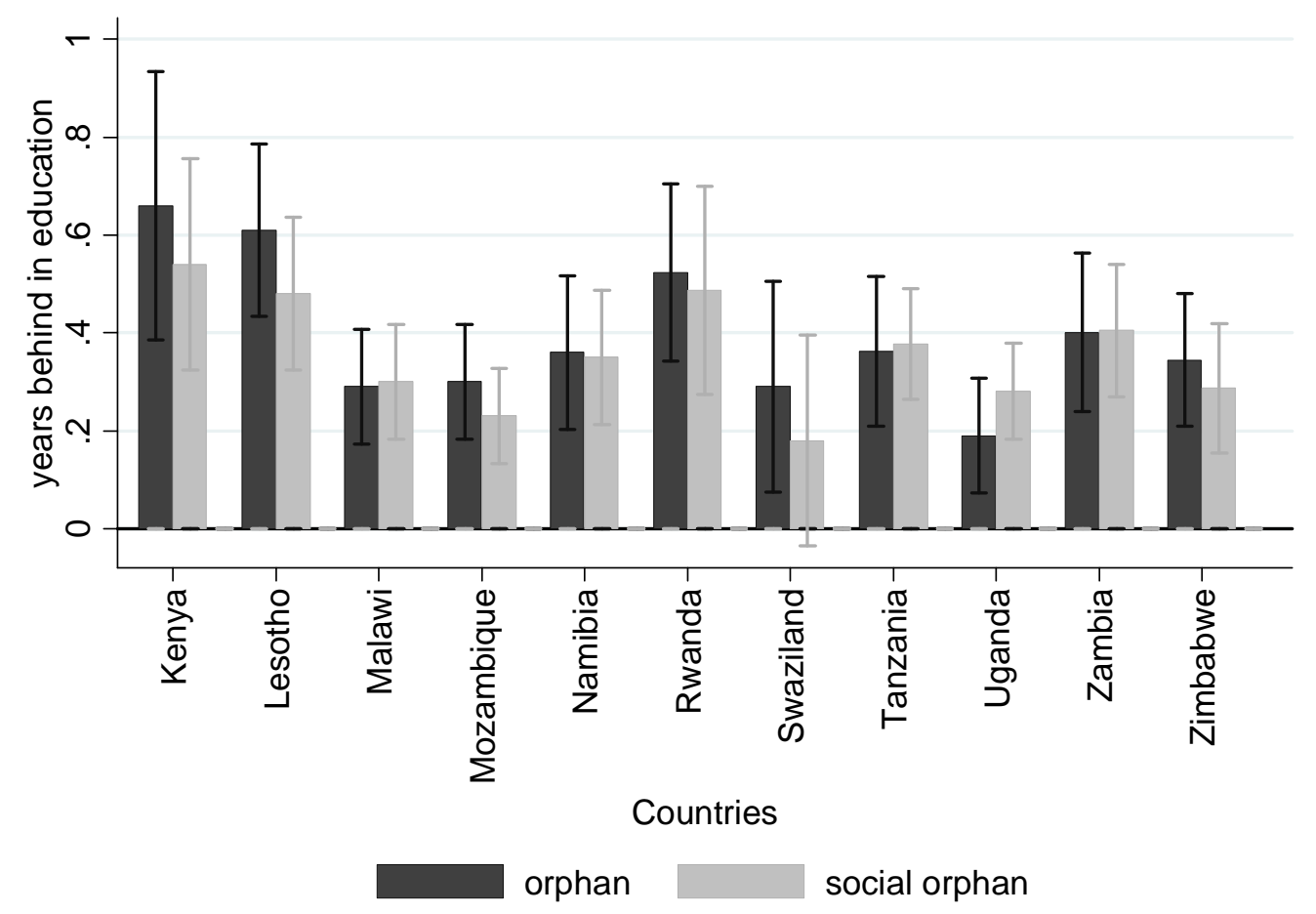

Note: Household fixed-effects regressions include control variables for age, age squared and gender (bars = coefficients, lines $=95 \%$ confidence bounds). Estimated separately by country. Specification 2 refers to binary variables indicating orphanhood and social orphanhood (reference category: living with mother and father). The point estimates underlying Figure 4 are presented in Table A 2 in the Appendix.

Source: Demographic and Health Survey (DHS): 2003-2007. Sample restricted to children aged 6/7-14. Own estimations.

Figure 4 expands this analysis to the group of social orphans (specification 2). Note that the reference categories to which we compare the orphans are different in Figure 4 and Figure 3. While we compare orphans to all other children in Figure 3, the reference category in Figure 4 excludes social orphans. Compared to children whose parents are alive and live with their biological parents, orphans significantly lag behind in education in all countries. The average effect size varies from somewhat more than half a year (Kenya, Lesotho and Rwanda) to one-fifth of a year in Uganda. Interestingly, social orphans are nearly as worse off in terms of their education as orphans. In Malawi, Tanzania and Uganda the point estimates are even higher for social orphans than for orphans. ${ }^{11}$

\footnotetext{
${ }^{11}$ The Bravais-Pearson correlation coefficient between the size of the orphan effect and the rate of orphanhood at the country level is 0.25 (based on the second specification of the fixed-effects regression). Interestingly, the correlation between the social orphan effect and the rate of social orphanhood is -0.55 . In other words, there is a weak tendency that social orphans are better off in countries with high rates of social orphanhood. This might be
} 
Figure 5: Fixed-effects estimates (specification 3): Educational outcomes

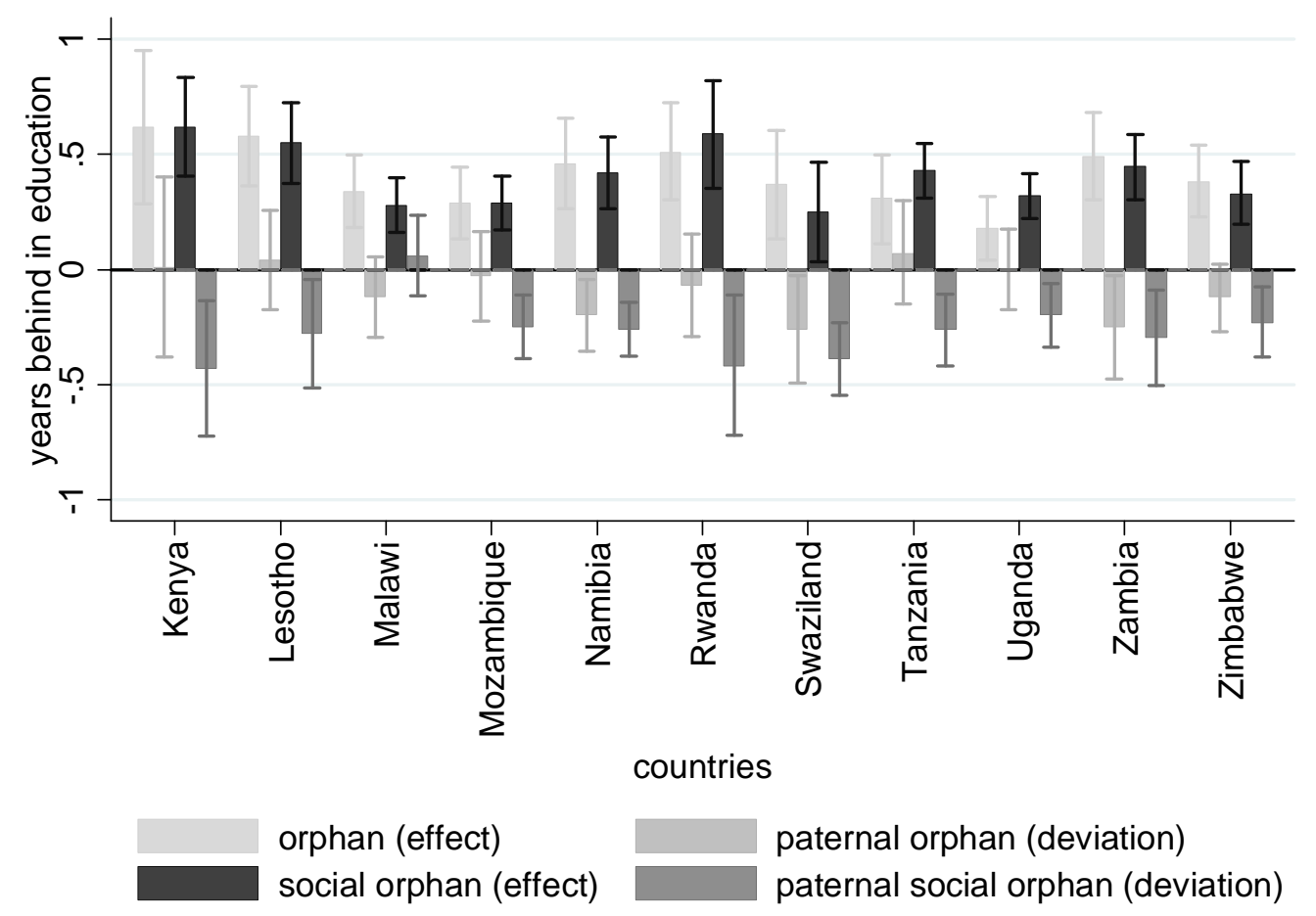

Note: Household fixed-effects regressions include control variables for age, age squared and gender (bars = coefficients, lines $=95 \%$ confidence bounds). Estimated separately by country. Specification 3 refers to binary variables indicating orphanhood, paternal orphanhood, social orphanhood, and paternal social orphanhood (reference category: living with mother and father). The point estimates underlying Figure 5 are presented in Table A 2 in the Appendix.

Source: Demographic and Health Survey (DHS): 2003-2007. Sample restricted to children aged 6/7-14. Own estimations.

In Figure 5 we further differentiate the analysis to children not growing up with a father (specification 3). Note that Figure 5 displays the absolute average effects of being an orphan or social orphan as compared to children living with both parents, while the paternal effects are additional effects for children when the father is dead or not living in the household. In most countries, we find that the additional effect of being a paternal orphan as compared to being an orphan whose mother died is zero. For Namibia, Swaziland, Zambia and Zimbabwe the effects are negative and significant. For social orphans, the additional effects are negative and significant (with the exception of an insignificant effect for Malawi). That means that these (social) orphans are worse off if they grow up without a mother as compared to growing up without a father. This additional negative effect is especially pronounced in countries with 
a high overall orphan effect, such as Kenya, Lesotho and Rwanda, but also Swaziland (where the orphan effect is also relatively high if we only consider orphans not living with a mother). In sum, the results demonstrate that children are specifically harmed with respect to their education if the mother is missing from the household.

Figure 6: Fixed-effects estimates (specification 1): Height and weight for age

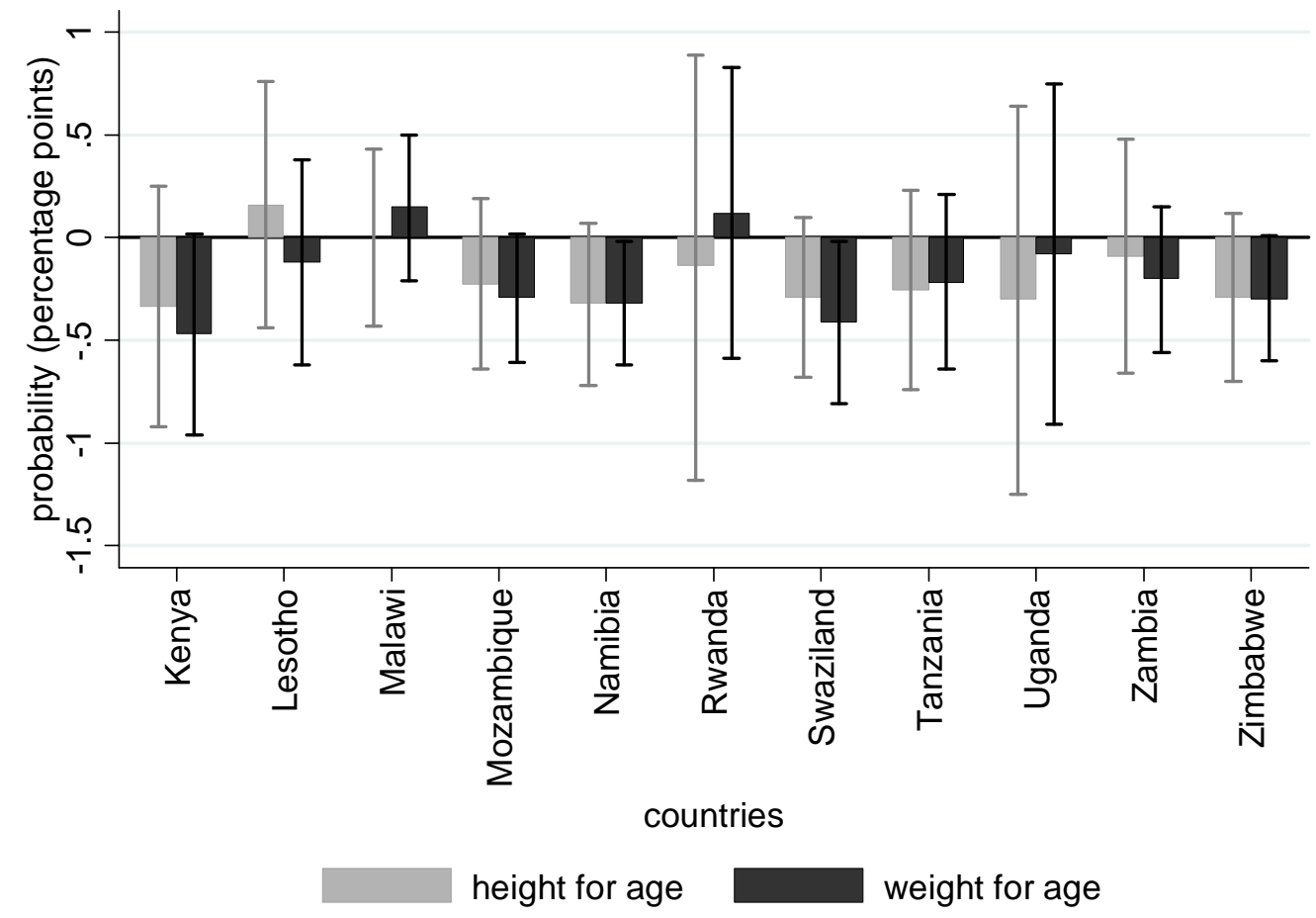

Note: Household fixed-effects regressions includes control variable for gender (bars $=$ coefficients, lines $=95 \%$ confidence bounds). Linear probability models, estimated separately by country. Specification 1 refers to a binary variable indicating orphanhood (reference category: at least one parent is alive). The point estimates underlying Figure 6 are presented in Table A 3 in the Appendix.

Source: Demographic and Health Survey (DHS): 2003-2007. Sample restricted to children aged 0-4. Own estimations.

One might ask whether the effect of being an orphan is less pronounced among children who lost their parents several years ago and have been living in blended households for a relatively longer period. In order to address this question, we repeated the above estimations with a subsample of "older orphans" by dropping all children living in households in which at least one child is aged six or younger (thus indicating that the parent died less than seven years ago, cf. 
Section 4). The results are robust with this sample. ${ }^{12}$ This suggests that the orphan effect is stable over time, i.e. that it is persistent even after orphans have been living in a blended household for several years. Because of this robustness, we do not present the respective results in more detail.

In Figure 6 to Figure 11, we present the orphan effect for children aged four and younger with respect to health outcomes. The point estimates presented in Figure 6 suggest that orphans have worse health outcomes than non-orphans in most countries: Orphans are relatively smaller and lighter for their age than non-orphans (except for a positive height estimate in Lesotho and positive weight estimates in Malawi and Rwanda).

Figure 7: Fixed-effects estimates (specification 1): Being stunted and underweight

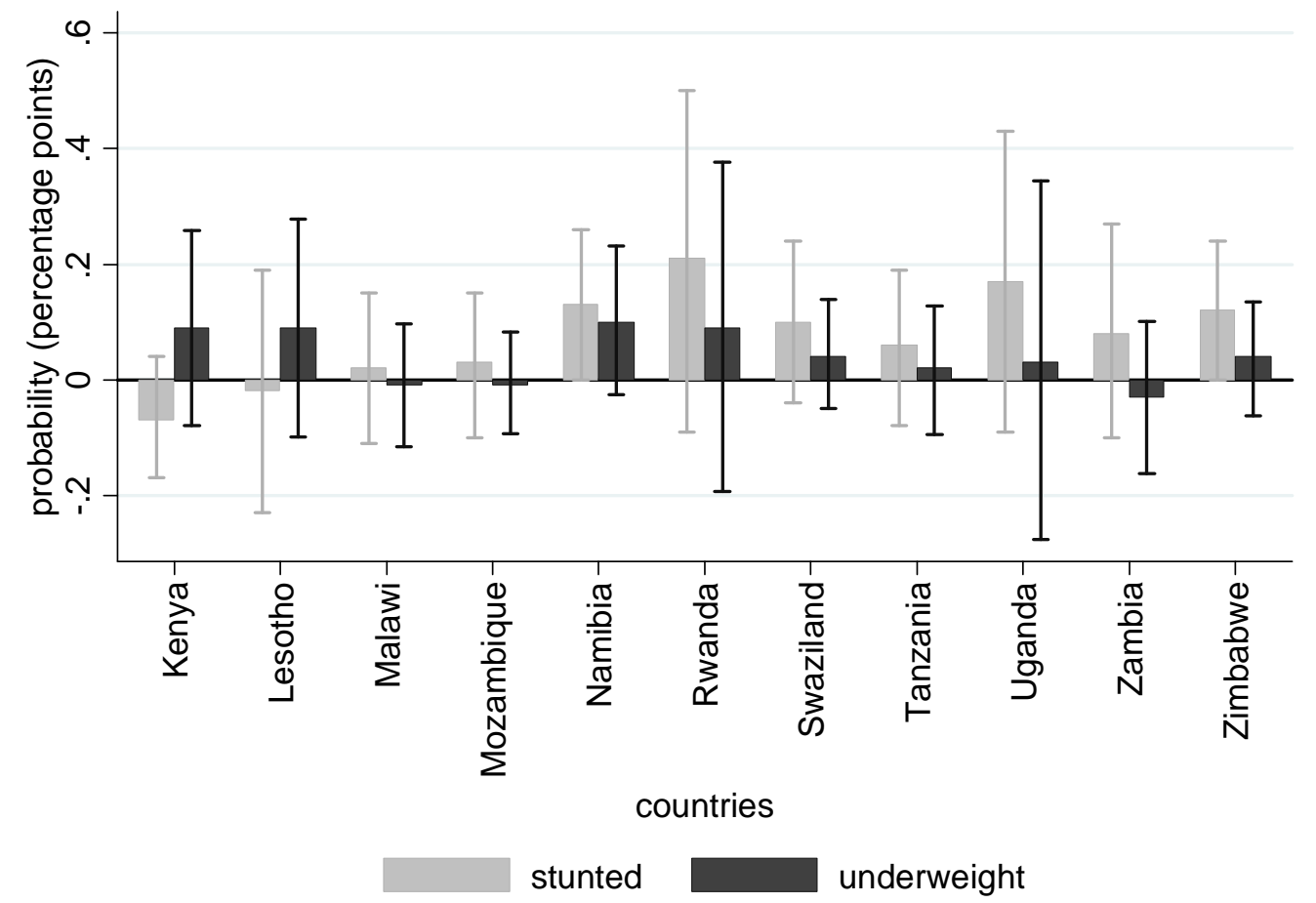

Note: Household fixed-effects regressions includes control variable gender (bars $=$ coefficients, lines $=95 \%$ confidence bounds). Linear probability models, estimated separately by country. Specification 1 refers to a binary variable indicating orphanhood (reference category: at least one parent is alive). The point estimates underlying Figure 7 are presented in Table A 3 in the Appendix.

Source: Demographic and Health Survey (DHS): 2003-2007. Sample restricted to children aged 0-4. Own estimations.

\footnotetext{
${ }^{12}$ All results are available upon request from the authors.
} 
Probably due to the relatively small sample sizes together with less variation within households as a result of a smaller proportion of orphans, these effects are often not statistically significant. However, the point estimates suggest a clear pattern. ${ }^{13}$ This is also reflected in Figure 7 where we consider the rather extreme health outcomes of being stunted and being underweight. According to the point estimates, orphans are more likely to be stunted and underweight in most countries. The orphan effect for stunted growth is strongest and statistically significant in Namibia (13 percentage points) and Zimbabwe (12 percentage points). However, the orphan effects for being underweight are not statistically significant in this specification. ${ }^{14}$

Figure 8: Fixed-effects estimates (specification 2): Height for age

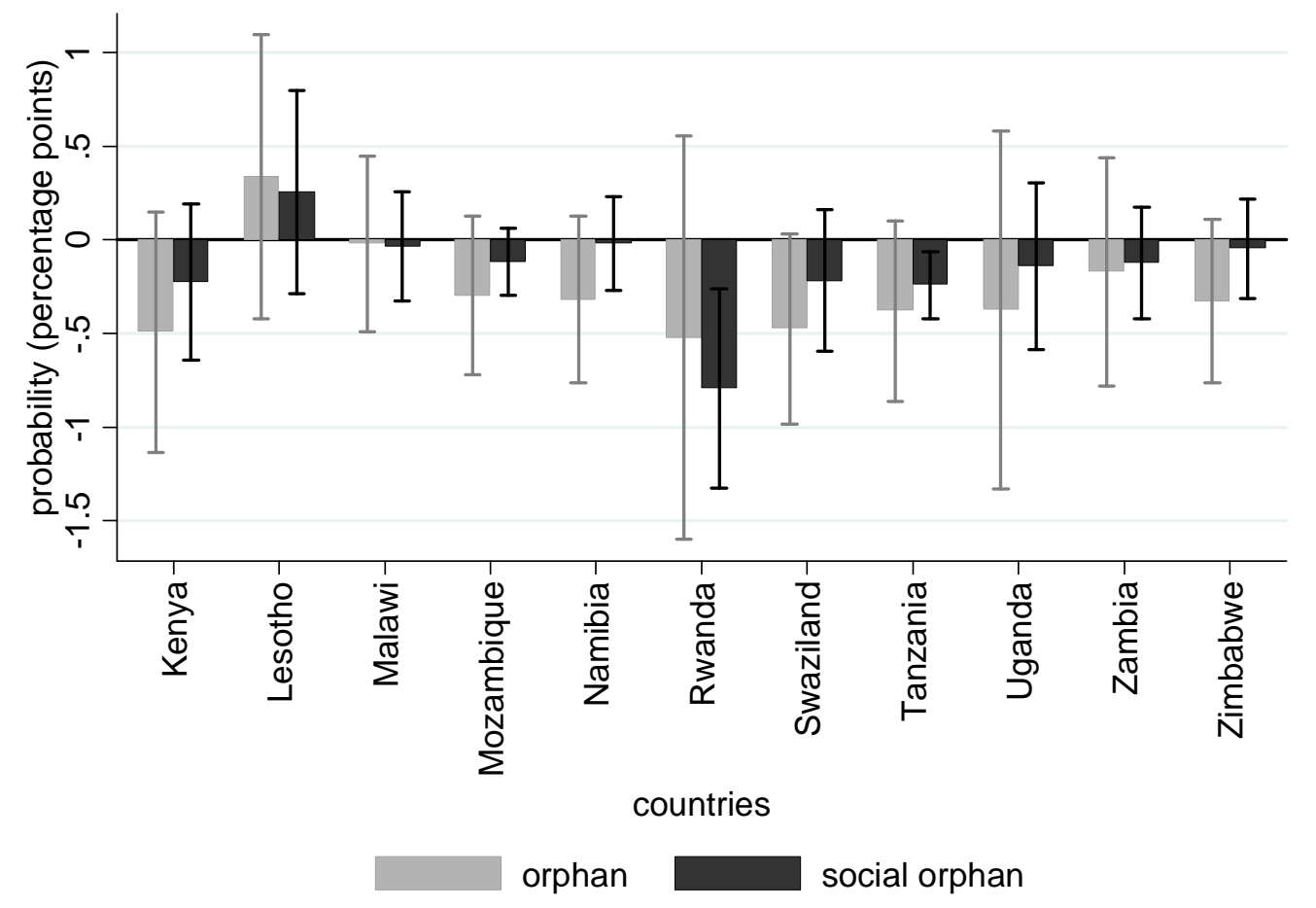

Note: Household fixed-effects regressions includes control variable for gender (bars $=$ coefficients, lines $=95 \%$ confidence bounds). Linear probability models, estimated separately by country. Specification 2 refers to binary variables indicating orphanhood and social orphanhood (reference category: living with both parents). The point estimates underlying this figure are presented in Table A 4 in the Appendix.

Source: Demographic and Health Survey (DHS): 2003-2007. Sample restricted to children aged 0-4. Own estimations.

\footnotetext{
${ }^{13}$ All effect sizes and standard errors are shown in Tables A3 and A4 the Appendix. The sample sizes have been presented in Table 1.

${ }^{14}$ If we look at children whose weight-for-age is below one standard deviation of the WHO reference population, we find more pronounced effects: Orphans are significantly more often below one standard deviation in Swaziland (26 percentage points), Lesotho (23 percentage points), Kenya (19 percentage points), Namibia (15 percentage points), and Zimbabwe (11 percentage points).
} 
Figure 9: Fixed-effects estimates (specification 2): Weight for age

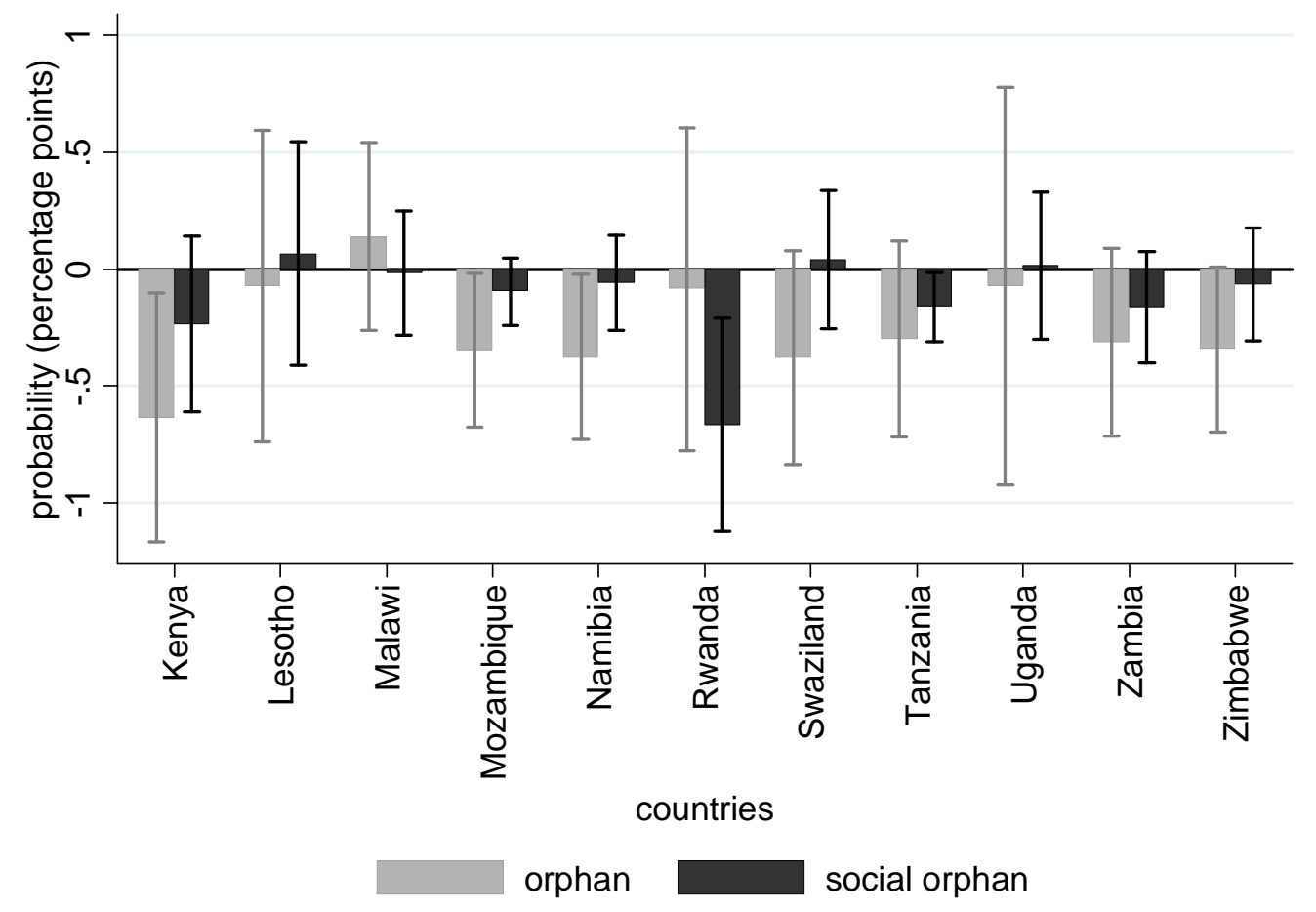

Note: Household fixed-effects regressions includes control variable for gender (bars = coefficients, lines $=95 \%$ confidence bounds). Linear probability models, estimated separately by country. Specification 2 refers to binary variables indicating orphanhood and social orphanhood (reference category: living with both parents). The point estimates underlying this figure are presented in Table A 4 in the Appendix.

Source: Demographic and Health Survey (DHS): 2003-2007. Sample restricted to children aged 0-4. Own estimations.

Figures 8-11 show all health outcomes for the fixed-effects regression specification that considers both orphans and social orphans. These figures demonstrate that orphans and social orphans are similarly affected in their health outcomes. In this comparison, the absolute effect size is predominantly higher for orphans. One exception is Rwanda, where the (statistically significant) estimates suggest that social orphans are smaller and lighter for their age than non-orphans.

Based on our second specification, we find significant growth-stunting effects for orphans in Namibia (14 percentage points), Uganda (23 percentage points) and Zimbabwe (14 percentage points) and for social orphans in Kenya (11 percentage points), Mozambique (6 percentage points), Rwanda (19 percentage points) and Tanzania (9 percentage points). 
Figure 10: Fixed-effects estimates (specification 2): Being stunted

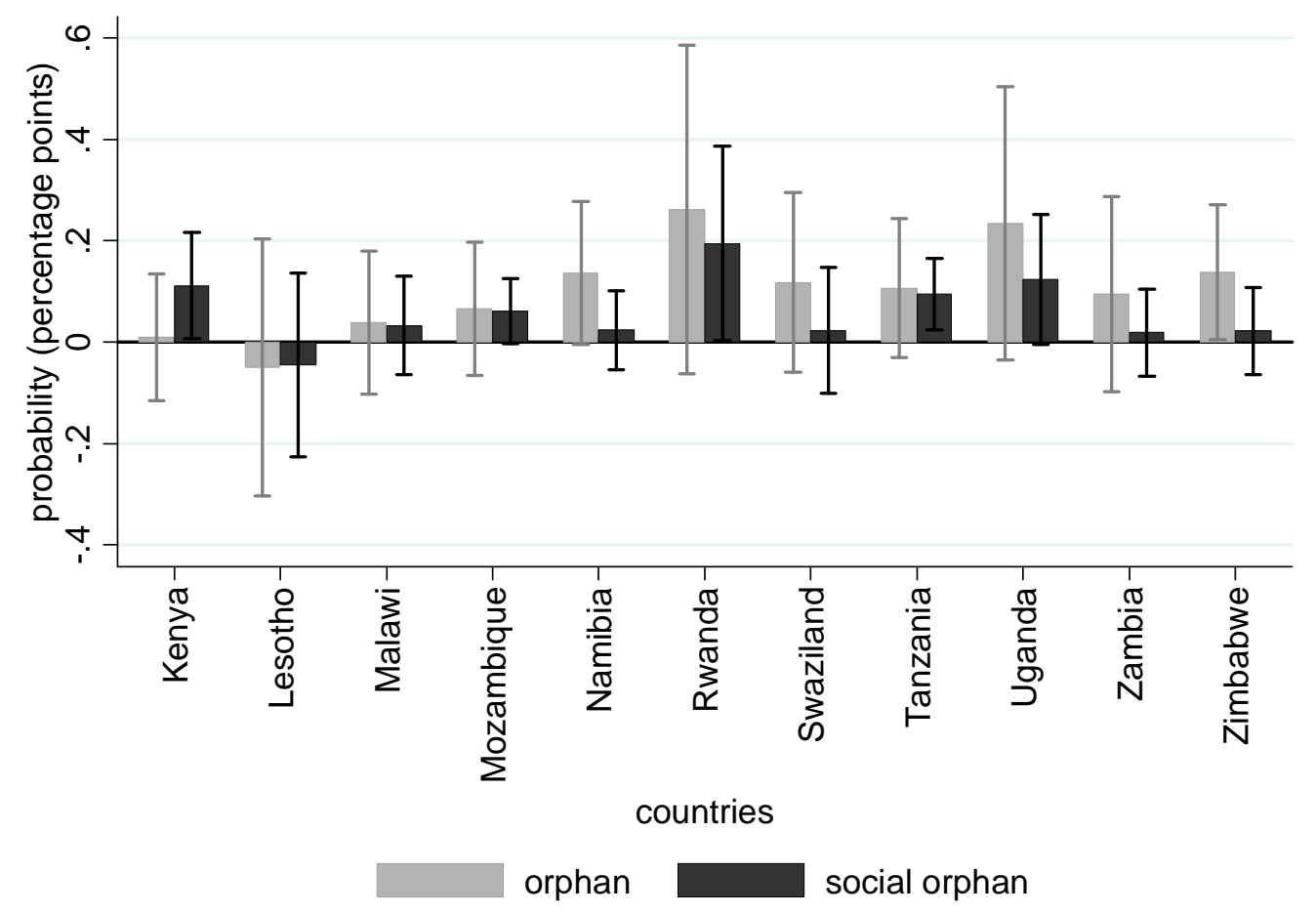

Note: Household fixed-effects regressions includes control variable for gender (bars = coefficients, lines $=95 \%$ confidence bounds). Linear probability models, estimated separately by country. Specification 2 refers to binary variables indicating orphanhood and social orphanhood (reference category: living with both parents). The point estimates underlying this figure are presented in Table A 4 in the Appendix.

Source: Demographic and Health Survey (DHS): 2003-2007. Sample restricted to children aged 0-4. Own estimations.

There are also significant effects on the incidence of being underweight in some countries:

When compared to the group of non-orphans and non-social orphans, orphans are

significantly more likely to be underweight in Kenya (19 percentage points) and Namibia (12

percentage points). For social orphans we find statistically significant effects for being

underweight in Kenya (15 percentage points) and Rwanda (18 percentage points). In sum, the

point estimates suggest that the nutritional status of both orphans and social orphans

compared to non-orphans is relatively poor in most of the considered countries. 
Figure 11: Fixed-effects estimates (specification 2): Being underweight

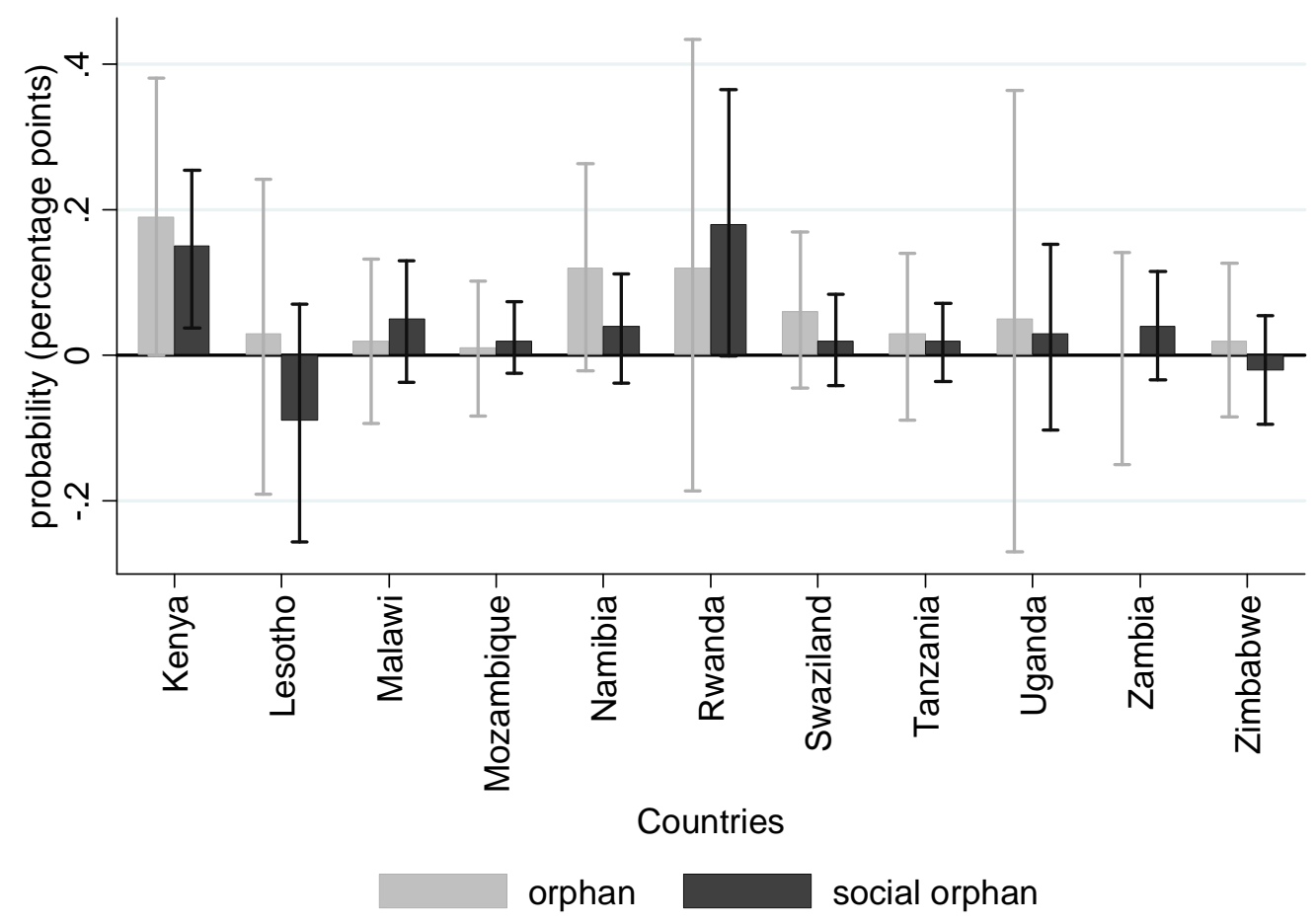

Note: Household fixed-effects regressions includes control variable for gender (bars $=$ coefficients, lines $=95 \%$ confidence bounds). Linear probability models, estimated separately by country. Specification 2 refers to binary variables indicating orphanhood and social orphanhood (reference category: living with both parents). The point estimates underlying this figure are presented in Table A 4 in the Appendix.

Source: Demographic and Health Survey (DHS): 2003-2007. Sample restricted to children aged 0-4. Own estimations.

\section{Discussion}

Our fixed-effects results imply that orphans living in blended households are worse off than non-orphans growing up under the same living condition. These orphans - as well as social orphans - lag behind in terms of years of education. In most countries, it seems that orphans and social orphans are also more often affected by extreme health outcomes related to malnutrition. The findings call for policies that specifically address the situation faced by orphans in sub-Saharan African countries. Regarding educational outcomes, we have also shown that social orphans are especially disadvantaged if they grow up without a mother. Therefore, policy measures should seek to specifically assist children whose mothers are alive, but absent from the household. 
Our results highlight the need for programs that seek to promote orphan education and health. One of the UN Millennium Development Goals is to "ensure that, by 2015, children everywhere [...] will be able to complete a full course of primary schooling” (Target 2). A further goal is related to child health (Target 4). The relatively poor situation of orphans with respect to both education and health is a serious obstacle to reaching these goals. 


\section{References}

Ainsworth. M., K. Beegle and G. Koda (2005): The Impact of Adult Mortality and Parental Deaths on Primary Schooling in North-Western Tanzania, The Journal of Development Studies 41 (3), 412-439.

Ainsworth. M. and D. Filmer (2002): Poverty, AIDS, and Children's Schooling: A Targeting Dilemma, The World Bank, Policy Research Working Paper 2885.

Ardington, C. and M. Leibbrandt (2010): Orphanhood and Schooling in South Africa: Trends in the vulnerability of orphans between 1993 and 2005, Economic Development and Cultural Change 58 (3), 507-536.

Atwine, B., E. Cantor-Graae and F. Bajunirwe (2005): Psychological distress among AIDS orphans in rural Uganda, Social Science \& Medicine 61(3), 555-564.

Beegle, K., D. Filmer, A. Stokes and L. Tiererova (2009): Orphanhood and the Living Arrangements of Children in Sub-Saharan Africa, World Bank Development Research Paper No. 4889.

Case, A. and C. Ardington (2006): The Impact of Parental Death on School Outcomes: Longitudinal Evidence from South Africa, Demography 43(3), 401-420.

Case, A., Paxson, Ch., J. Ableidinger (2004): Orphans in Africa: Parental Death, Poverty, and School Enrollment, Demography 41(3), 483-508.

Deleire, T. and A. Kalil (2002): Good Things Come in Threes: Single-Parent Multigenerational Family Structure and Adolescent Adjustment, Demography 39(2), 393413.

Evans, D. and E. Miguel (2007): Orphans and Schooling in Africa: A Longitudinal Analysis, Demography 44 (1), 35-57.

Gunderson, T. and C. Kelly (2008): School Attendance and Educational Enrollment for Maternal Orphans in Zimbabwe: An Instrumental Variables Approach, Journal of African Development 10 (1), 33-50.

Hamilton, W.D. (1964): The Genetical Evolution of Social Behavior, Journal of Theoretical Biology 7(1), 1-16.

Heymann, J., A. Earle, D. Rajaraman, C. Miller and K. Bogen (2007): Extended Family Caring for Children Orphaned by AIDS: Balancing Essential Work and Caregiving in a High HIV Prevalence Nation, AIDS Care, 19 (3), 337-345.

Lorentzen, P., J. McMillan and R. Wacziarg (2008): Death and Development, Journal of Economic Growth 13, 81-124.

Lloyd, C.B. and P.C. Hewett (2003): Primary Schooling in sub-Saharan Africa: Recent Trends and Current Challenges, Population Council Working Papers 176. 
Mirumba, S. (2005): The Impact of the Southern and Eastern Africa Consortium for Monitoring Educational Quality (SACMEQ), Prospects, 35 (1), 91-108.

Monarsch, R. and T. Boerna (2004): Orphanhood and Childcare Patterns in sub-Saharan Africa: An Analysis of National Surveys from 40 Countries, AIDS 18(2), 55-65.

The World Bank / UNICEF (2009): Abolishing School Fees in Africa, Development Practice in Education, http://www.unicef.org/publications/files/Aboloshing_School_Fees_in_Africa.pdf (retrieved November 2010).

UNDAIDS (2010): 2010 Report on the global AIDS epidemic, http://www.unaids.org/globalreport/Global_report.htm (retrieved December 2010).

UNICEF (2006): Africa's Orphaned and Vulnerable Generations Children Affected by AIDS, http://www.unicef.org/publications/files/Africas_Orphaned_and_Vulnerable_Generations _Children_Affected_by_AIDS.pdf (retrieved November 2010).

USAID (2010): School Fee Abolition: Impact on Learning and Persistence, http://www.equip123.net/docs/E2-School_Fees_Learning_and_Persistence-IB.pdf (retrieved November 2010). 


\section{Appendix}

Table A 1: Proportion of orphans and social orphans among children aged 0-14 in the DHS data (in \%)

\begin{tabular}{lccccc}
\hline Country, year & orphans & $\begin{array}{c}\text { paternal } \\
\text { orphans }\end{array}$ & $\begin{array}{c}\text { social } \\
\text { orphans }\end{array}$ & $\begin{array}{c}\text { paternal } \\
\text { social } \\
\text { orphans }\end{array}$ & observations \\
\hline Kenya, 2003 & 11.05 & 7.22 & 29.08 & 19.75 & 15,825 \\
Lesotho, 2004 & 26.47 & 18.28 & 24.61 & 10.22 & 14,309 \\
Malawi, 2004 & 13.24 & 7.79 & 27.47 & 14.91 & 29,137 \\
Mozambique, 2003 & 10.64 & 6.79 & 30.69 & 18.49 & 28,901 \\
Namibia, 2006-7 & 14.95 & 9.08 & 57.72 & 28.88 & 16,440 \\
Rwanda, 2005 & 17.57 & 11.70 & 19.36 & 12.43 & 21,628 \\
Swaziland, 2006-7 & 20.39 & 12.32 & 56.15 & 32.31 & 9,299 \\
Tanzania (mainland), 2004-5 & 8.55 & 5.40 & 29.78 & 16.48 & 17,839 \\
Tanzania (all regions), 2004-5 & 7.94 & 5.12 & 29.53 & 15.32 & 22,683 \\
Uganda, 2006 & 13.53 & 7.95 & 31.46 & 16.04 & 23,547 \\
Zambia, 2007 & 13.03 & 7.52 & 28.43 & 15.22 & 17,302 \\
Zimbabwe, 2005-6 & 21.54 & 13.02 & 36.23 & 19.80 & 18,422 \\
\hline \hline
\end{tabular}

Note: Table A1 presents the unweighted percentages from the DHS data which are displayed in Figure 1 and Figure 2.

Source: Demographic and Health Survey (DHS): 2003-2007. Own calculations. 
Table A 2: Fixed-effects estimates of orphan indicators - Outcome: Years lagging behind in education

\begin{tabular}{|c|c|c|c|c|c|c|c|}
\hline \multirow[b]{2}{*}{ Country, year } & \multirow{2}{*}{$\begin{array}{c}\text { Specification 1: } \\
\text { Reference } \\
\text { group }= \\
\text { non-orphans } \\
\text { orphans }\end{array}$} & \multicolumn{2}{|c|}{$\begin{array}{c}\text { Specification 2: } \\
\text { Reference group = } \\
\text { children living with } \\
\text { mother and father }\end{array}$} & \multicolumn{4}{|c|}{$\begin{array}{c}\text { Specification } 3 \\
\text { Reference group = } \\
\text { children living with } \\
\text { mother and father }\end{array}$} \\
\hline & & orphans & $\begin{array}{c}\text { social } \\
\text { orphans }\end{array}$ & orphans & $\begin{array}{l}\text { paternal } \\
\text { orphans }\end{array}$ & $\begin{array}{c}\text { social } \\
\text { orphans }\end{array}$ & $\begin{array}{c}\text { paternal } \\
\text { social } \\
\text { orphans }\end{array}$ \\
\hline $\begin{array}{l}\text { Kenya, } \\
2003\end{array}$ & $\begin{array}{c}0.39 * * * \\
(0.12)\end{array}$ & $\begin{array}{c}0.66^{* * *} \\
(0.14)\end{array}$ & $\begin{array}{c}0.54 * * * \\
(0.11)\end{array}$ & $\begin{array}{c}0.62 * * * \\
(0.17)\end{array}$ & $\begin{array}{c}0.01 \\
(0.20)\end{array}$ & $\begin{array}{c}0.62 * * * \\
(0.11)\end{array}$ & $\begin{array}{c}-0.43^{* * * *} \\
(0.15)\end{array}$ \\
\hline $\begin{array}{l}\text { Lesotho, } \\
2004\end{array}$ & $\begin{array}{c}0.35 * * * \\
(0.07)\end{array}$ & $\begin{array}{c}0.61^{* * *} \\
(0.09)\end{array}$ & $\begin{array}{c}0.48 * * * \\
(0.08)\end{array}$ & $\begin{array}{c}0.58 * * * \\
(0.11)\end{array}$ & $\begin{array}{c}0.04 \\
(0.11)\end{array}$ & $\begin{array}{c}0.55^{* * *} \\
(0.09)\end{array}$ & $\begin{array}{c}-0.28 * * \\
(0.12)\end{array}$ \\
\hline $\begin{array}{l}\text { Malawi, } \\
2004\end{array}$ & $\begin{array}{c}0.13^{* *} \\
(0.06)\end{array}$ & $\begin{array}{c}0.29 * * * \\
(0.06)\end{array}$ & $\begin{array}{c}0.30 * * * \\
(0.06)\end{array}$ & $\begin{array}{c}0.34 * * * \\
(0.08)\end{array}$ & $\begin{array}{l}-0.12 \\
(0.09)\end{array}$ & $\begin{array}{c}0.28 * * * \\
(0.06)\end{array}$ & $\begin{array}{c}0.06 \\
(0.09)\end{array}$ \\
\hline $\begin{array}{l}\text { Mozambique, } \\
2003\end{array}$ & $\begin{array}{c}0.17 * * * \\
(0.06)\end{array}$ & $\begin{array}{c}0.30 * * * \\
(0.06)\end{array}$ & $\begin{array}{c}0.23^{* * *} \\
(0.05)\end{array}$ & $\begin{array}{c}0.29 * * * \\
(0.08)\end{array}$ & $\begin{array}{l}-0.03 \\
(0.10)\end{array}$ & $\begin{array}{c}0.29 * * * \\
(0.06)\end{array}$ & $\begin{array}{c}-0.25 * * * \\
(0.07)\end{array}$ \\
\hline $\begin{array}{l}\text { Namibia, } \\
\text { 2006-7 }\end{array}$ & $\begin{array}{c}0.06 \\
(0.05)\end{array}$ & $\begin{array}{c}0.36 * * * \\
(0.08)\end{array}$ & $\begin{array}{c}0.35 * * * \\
(0.07)\end{array}$ & $\begin{array}{c}0.46 * * * \\
(0.10)\end{array}$ & $\begin{array}{c}-0.20 * * \\
(0.08)\end{array}$ & $\begin{array}{c}0.42^{* * *} \\
(0.08)\end{array}$ & $\begin{array}{c}-0.26 * * * \\
(0.06)\end{array}$ \\
\hline $\begin{array}{l}\text { Rwanda, } \\
2005\end{array}$ & $\begin{array}{c}0.28 * * * \\
(0.08)\end{array}$ & $\begin{array}{c}0.52 * * * \\
(0.09)\end{array}$ & $\begin{array}{c}0.49 * * * \\
(0.11)\end{array}$ & $\begin{array}{c}0.51 * * * \\
(0.11)\end{array}$ & $\begin{array}{l}-0.07 \\
(0.11)\end{array}$ & $\begin{array}{c}0.59 * * * \\
(0.12)\end{array}$ & $\begin{array}{c}-0.42 * * * \\
(0.16)\end{array}$ \\
\hline $\begin{array}{l}\text { Swaziland, } \\
\text { 2006-7 }\end{array}$ & $\begin{array}{c}0.14 * * \\
(0.07)\end{array}$ & $\begin{array}{c}0.29 * * \\
(0.11)\end{array}$ & $\begin{array}{l}0.18 * \\
(0.11)\end{array}$ & $\begin{array}{c}0.37 * * * \\
(0.12)\end{array}$ & $\begin{array}{c}-0.26 * * \\
(0.12)\end{array}$ & $\begin{array}{l}0.25 * \\
(0.11)\end{array}$ & $\begin{array}{c}-0.39 * * * \\
(0.08)\end{array}$ \\
\hline $\begin{array}{l}\text { Tanzania, } \\
\text { 2004-5 }\end{array}$ & $\begin{array}{c}0.13 * \\
(0.07)\end{array}$ & $\begin{array}{c}0.36 * * * \\
(0.08)\end{array}$ & $\begin{array}{c}0.38 * * * \\
(0.06)\end{array}$ & $\begin{array}{c}0.31 * * * \\
(0.10)\end{array}$ & $\begin{array}{c}0.07 \\
(0.11)\end{array}$ & $\begin{array}{c}0.43^{* * *} \\
(0.06)\end{array}$ & $\begin{array}{c}-0.26 * * * \\
(0.08)\end{array}$ \\
\hline $\begin{array}{l}\text { Uganda, } \\
2006\end{array}$ & $\begin{array}{c}0.02 \\
(0.05)\end{array}$ & $\begin{array}{c}0.19 * * * \\
(0.06)\end{array}$ & $\begin{array}{c}0.28 * * * \\
(0.05)\end{array}$ & $\begin{array}{c}0.18 * * \\
(0.07)\end{array}$ & $\begin{array}{c}0.00 \\
(0.09)\end{array}$ & $\begin{array}{c}0.32 * * * \\
(0.05)\end{array}$ & $\begin{array}{c}-0.20 * * * \\
(0.07)\end{array}$ \\
\hline $\begin{array}{l}\text { Zambia, } \\
2007\end{array}$ & $\begin{array}{c}0.18^{* *} \\
(0.07)\end{array}$ & $\begin{array}{c}0.40 * * * \\
(0.08)\end{array}$ & $\begin{array}{c}0.40 * * * \\
(0.07)\end{array}$ & $\begin{array}{c}0.49 * * * \\
(0.10)\end{array}$ & $\begin{array}{c}-0.25^{* *} \\
(0.11)\end{array}$ & $\begin{array}{c}0.45^{* * *} \\
(0.07)\end{array}$ & $\begin{array}{c}-0.30 * * * \\
(0.11)\end{array}$ \\
\hline $\begin{array}{l}\text { Zimbabwe, } \\
2005-6\end{array}$ & $\begin{array}{c}0.15 * * * \\
(0.05)\end{array}$ & $\begin{array}{c}0.34^{* * *} \\
(0.07)\end{array}$ & $\begin{array}{c}0.29 * * * \\
(0.07)\end{array}$ & $\begin{array}{c}0.38 * * * \\
(0.08)\end{array}$ & $\begin{array}{l}-0.12^{*} \\
(0.07)\end{array}$ & $\begin{array}{c}0.33^{* * *} \\
(0.07)\end{array}$ & $\begin{array}{c}-0.23^{* * *} \\
(0.08)\end{array}$ \\
\hline
\end{tabular}

Note: Coefficients (standard errors) from household fixed-effects regressions including control variables for age, age squared and gender. Estimated separately by country. The shown estimates are coefficient estimates on binary family structure indicators: Specification 1 includes an indicator for orphanhood (cf. Figure 3). Specification 2 includes indicators for orphanhood and social orphanhood (cf. Figure 4). Specification 3 additionally includes indicators for paternal death and children not living with a father (cf. Figure 5).

Source: Demographic and Health Survey (DHS): 2003-2007. Sample restricted to children aged 6/7-14. Own estimations. 
Table A 3: Fixed-effects estimates of orphan indicator - Outcome: Child health

\begin{tabular}{lcccc}
\hline \hline Country, year & height & weight & stunted & underweight \\
\hline Kenya, & -0.34 & $-0.47^{*}$ & -0.07 & 0.09 \\
2003 & $(0.30)$ & $(0.25)$ & $(0.05)$ & $(0.09)$ \\
Lesotho, & 0.16 & -0.12 & -0.02 & 0.09 \\
2004 & $(0.30)$ & $(0.25)$ & $(0.11)$ & $(0.10)$ \\
Malawi, & 0.00 & 0.15 & 0.02 & -0.01 \\
2004 & $(0.22)$ & $(0.18)$ & $(0.07)$ & $(0.05)$ \\
Mozambique, & -0.23 & $-0.29^{*}$ & 0.03 & -0.01 \\
2003 & $(0.21)$ & $(0.16)$ & $(0.06)$ & $0.04)$ \\
Namibia, & -0.32 & $-0.32^{* *}$ & $0.13^{* *}$ & 0.10 \\
2006-7 & $(0.20)$ & $(0.15)$ & $(0.06)$ & $(0.07)$ \\
Rwanda, & -0.14 & 0.12 & 0.21 & 0.09 \\
2005 & $(0.53)$ & $(0.36)$ & $(0.15)$ & $(0.15)$ \\
Swaziland, & -0.29 & $-0.41^{* *}$ & 0.10 & 0.04 \\
2006-7 & $(0.20)$ & $(0.20)$ & $(0.07)$ & $(0.05)$ \\
Tanzania, & -0.26 & -0.22 & 0.06 & 0.02 \\
2004-5 & $(0.25)$ & $(0.21)$ & $(0.07)$ & $(0.06)$ \\
Uganda, & -0.30 & -0.08 & 0.17 & 0.03 \\
2006 & $(0.48)$ & $(0.42)$ & $(0.13)$ & $(0.16)$ \\
Zambia, & -0.09 & -0.20 & 0.08 & -0.03 \\
2007 & $(0.29)$ & $(0.18)$ & $(0.09)$ & $(0.07)$ \\
Zimbabwe, & -0.29 & $-0.30^{*}$ & $0.12^{* *}$ & 0.04 \\
2005-6 & $(0.21)$ & $(0.16)$ & $(0.06)$ & $(0.05)$ \\
\hline \hline
\end{tabular}

Note: Coefficients (standard errors) from household fixed-effects regressions including control variables for age, age squared and gender. Linear probability models, estimated separately by country. The shown estimates are coefficient estimates on a binary orphan indicator (Specification 1, cf. Figure 6 and Figure 7)

Source: Demographic and Health Survey (DHS): 2003-2007. Sample restricted to children aged 6/7-14. Own estimations. 
Table A 4: Fixed-effects estimates of orphan and social orphan indicator - Outcome: Child health

\begin{tabular}{|c|c|c|c|c|c|c|c|c|}
\hline \multirow[b]{2}{*}{ Country, year } & \multicolumn{2}{|c|}{ height } & \multicolumn{2}{|c|}{ weight } & \multicolumn{2}{|c|}{ stunted } & \multicolumn{2}{|c|}{ underweight } \\
\hline & orphan & $\begin{array}{c}\text { social } \\
\text { orphan }\end{array}$ & orphan & $\begin{array}{c}\text { social } \\
\text { orphan }\end{array}$ & orphan & $\begin{array}{c}\text { social } \\
\text { orphan }\end{array}$ & orphan & $\begin{array}{l}\text { social } \\
\text { orphan }\end{array}$ \\
\hline $\begin{array}{l}\text { Kenya, } \\
2003\end{array}$ & $\begin{array}{c}-0.49 \\
(0.33)\end{array}$ & $\begin{array}{c}-0.23 \\
(0.21)\end{array}$ & $\begin{array}{c}-0.64^{* *} \\
(0.27)\end{array}$ & $\begin{array}{c}-0.24 \\
(0.19)\end{array}$ & $\begin{array}{c}0.01 \\
(0.06)\end{array}$ & $\begin{array}{c}0.11 * * \\
(0.05)\end{array}$ & $\begin{array}{l}0.19 * \\
(0.10)\end{array}$ & $\begin{array}{c}0.15 * * \\
(0.06)\end{array}$ \\
\hline $\begin{array}{l}\text { Lesotho, } \\
2004\end{array}$ & $\begin{array}{c}0.34 \\
(0.38)\end{array}$ & $\begin{array}{c}0.25 \\
(0.28)\end{array}$ & $\begin{array}{c}-0.07 \\
(0.34)\end{array}$ & $\begin{array}{c}0.07 \\
(0.24)\end{array}$ & $\begin{array}{l}-0.05 \\
(0.13)\end{array}$ & $\begin{array}{c}-0.05 \\
(0.09)\end{array}$ & $\begin{array}{c}0.03 \\
(0.11)\end{array}$ & $\begin{array}{l}-0.09 \\
(0.08)\end{array}$ \\
\hline $\begin{array}{l}\text { Malawi, } \\
2004\end{array}$ & $\begin{array}{l}-0.02 \\
(0.24)\end{array}$ & $\begin{array}{c}-0.04 \\
(0.15)\end{array}$ & $\begin{array}{c}0.14 \\
(0.21)\end{array}$ & $\begin{array}{c}-0.02 \\
(0.14)\end{array}$ & $\begin{array}{c}0.04 \\
(0.07)\end{array}$ & $\begin{array}{c}0.03 \\
(0.05)\end{array}$ & $\begin{array}{c}0.02 \\
(0.06)\end{array}$ & $\begin{array}{c}0.0 .5 \\
(0.04)\end{array}$ \\
\hline $\begin{array}{l}\text { Mozambique, } \\
2003\end{array}$ & $\begin{array}{c}-0.30 \\
(0.22)\end{array}$ & $\begin{array}{c}-0.12 \\
(0.09)\end{array}$ & $\begin{array}{c}-0.35 * * \\
(0.17)\end{array}$ & $\begin{array}{l}-0.10 \\
(0.07)\end{array}$ & $\begin{array}{c}0.07 \\
(0.07)\end{array}$ & $\begin{array}{l}0.06 * \\
(0.03)\end{array}$ & $\begin{array}{c}0.01 \\
(0.05)\end{array}$ & $\begin{array}{c}0.02 \\
(0.03)\end{array}$ \\
\hline $\begin{array}{l}\text { Namibia, } \\
\text { 2006-7 }\end{array}$ & $\begin{array}{c}-0.32 \\
(0.23)\end{array}$ & $\begin{array}{c}-0.02 \\
(0.13)\end{array}$ & $\begin{array}{c}-0.38 * * \\
(0.18)\end{array}$ & $\begin{array}{l}-0.06 \\
(0.10)\end{array}$ & $\begin{array}{c}0.14^{*} \\
(0.07)\end{array}$ & $\begin{array}{c}0.02 \\
(0.04)\end{array}$ & $\begin{array}{l}0.12 * \\
(0.07)\end{array}$ & $\begin{array}{c}0.04 \\
(0.04)\end{array}$ \\
\hline $\begin{array}{l}\text { Rwanda, } \\
2005\end{array}$ & $\begin{array}{l}-0.52 \\
(0.55)\end{array}$ & $\begin{array}{c}-0.79 * * * \\
(0.27)\end{array}$ & $\begin{array}{l}-0.09 \\
(0.35)\end{array}$ & $\begin{array}{c}-0.67 * * * \\
(0.23)\end{array}$ & $\begin{array}{c}0.26 \\
(0.17)\end{array}$ & $\begin{array}{c}0.19 * * \\
(0.10)\end{array}$ & $\begin{array}{c}0.12 \\
(0.16)\end{array}$ & $\begin{array}{l}0.18^{*} \\
(0.09)\end{array}$ \\
\hline $\begin{array}{l}\text { Swaziland, } \\
\text { 2006-7 }\end{array}$ & $\begin{array}{c}-0.48 * \\
(0.26)\end{array}$ & $\begin{array}{c}-0.22 \\
(0.19)\end{array}$ & $\begin{array}{c}-0.38 \\
(0.23)\end{array}$ & $\begin{array}{c}0.04 \\
(0.15)\end{array}$ & $\begin{array}{c}0.12 \\
(0.09)\end{array}$ & $\begin{array}{c}0.02 \\
(0.06)\end{array}$ & $\begin{array}{c}0.06 \\
(0.05)\end{array}$ & $\begin{array}{c}0.02 \\
(0.03)\end{array}$ \\
\hline $\begin{array}{l}\text { Tanzania, } \\
\text { 2004-5 }\end{array}$ & $\begin{array}{c}-0.38 \\
(0.25)\end{array}$ & $\begin{array}{c}-0.24^{* * *} \\
(0.09)\end{array}$ & $\begin{array}{c}-0.30 \\
(0.21)\end{array}$ & $\begin{array}{c}-0.16 * * \\
(0.08)\end{array}$ & $\begin{array}{c}0.11 \\
(0.07)\end{array}$ & $\begin{array}{c}0.09 * * * \\
(0.04)\end{array}$ & $\begin{array}{c}0.03 \\
(0.06)\end{array}$ & $\begin{array}{c}0.02 \\
(0.03)\end{array}$ \\
\hline $\begin{array}{l}\text { Uganda, } \\
2006\end{array}$ & $\begin{array}{l}-0.37 \\
(0.49)\end{array}$ & $\begin{array}{c}-0.14 \\
(0.23)\end{array}$ & $\begin{array}{l}-0.07 \\
(0.43)\end{array}$ & $\begin{array}{c}0.01 \\
(0.16)\end{array}$ & $\begin{array}{l}0.23^{*} \\
(0.14)\end{array}$ & $\begin{array}{l}0.12^{*} \\
(0.07)\end{array}$ & $\begin{array}{c}0.05 \\
(0.16)\end{array}$ & $\begin{array}{c}0.03 \\
(0.07)\end{array}$ \\
\hline $\begin{array}{l}\text { Zambia, } \\
2007\end{array}$ & $\begin{array}{l}-0.17 \\
(0.31)\end{array}$ & $\begin{array}{c}-0.12 \\
(0.15)\end{array}$ & $\begin{array}{c}-0.31 \\
(0.20)\end{array}$ & $\begin{array}{c}-0.16 \\
(0.12)\end{array}$ & $\begin{array}{c}0.09 \\
(0.10)\end{array}$ & $\begin{array}{c}0.02 \\
(0.04)\end{array}$ & $\begin{array}{c}0.00 \\
(0.07)\end{array}$ & $\begin{array}{c}0.04 \\
(0.04)\end{array}$ \\
\hline $\begin{array}{l}\text { Zimbabwe, } \\
2005-6\end{array}$ & $\begin{array}{c}-0.33 \\
(0.22) \\
\end{array}$ & $\begin{array}{c}-0.05 \\
(0.14) \\
\end{array}$ & $\begin{array}{c}-0.34 * \\
(0.18) \\
\end{array}$ & $\begin{array}{c}-0.07 \\
(0.12) \\
\end{array}$ & $\begin{array}{c}0.14^{* *} \\
(0.07) \\
\end{array}$ & $\begin{array}{c}0.02 \\
(0.04) \\
\end{array}$ & $\begin{array}{c}0.02 \\
(0.05) \\
\end{array}$ & $\begin{array}{c}-0.02 \\
(0.04) \\
\end{array}$ \\
\hline
\end{tabular}

Note: Coefficients (standard errors) from household fixed-effects regressions including control variables for age, age squared and gender. Linear probability models, estimated separately by country. The shown estimates are coefficient estimates on binary indicators for being an orphan or social orphan (Reference: living with both parents), cf. Figure 8 - Figure 11 (Specification 2).

Source: Demographic and Health Survey (DHS): 2003-2007. Sample restricted to children aged 6/7-14. Own estimations. 